\title{
Pacific
}

Journal of

Mathematics

\section{ROTATIONALLY SYMMETRIC HYPERSURFACES WITH PRESCRIBED MEAN CURVATURE}

MARIE-FRANÇOISE BIDAUT-VÉRON 


\title{
ROTATIONALLY SYMMETRIC HYPERSURFACES WITH PRESCRIBED MEAN CURVATURE
}

\author{
Marie-Francoise Bidaut-Veron
}

Here we study the singular radial solutions of the prescribed mean curvature equation

$$
\operatorname{div} \frac{D u}{\sqrt{1+|D u|^{2}}}+f(u)=0, \quad \text { in } \mathbb{R}^{N} /\{0\}
$$

where $f$ is increasing and has the sign of $u$ near infinity. We prove the local existence of a generalized singular solution under slight growth assumptions on $f$. In the physical case $N=2$ we prove that the curve is asymptotic to the curve $r|f(u)|=1$. We also study the global behaviour of the solutions.

\section{Introduction.}

In this paper we consider the question of existence and behaviour of radial singular solutions of the prescribed mean curvature in $\mathbb{R}^{N}(N \geqq 2)$ :

$$
\operatorname{div}\left(\frac{D u}{\sqrt{1+|D u|^{2}}}\right)+f(u)=0 \quad \text { in } \mathbb{R}^{N} /\{0\}
$$

with

$$
u(x) \rightarrow-\infty \quad \text { as }\|x\| \rightarrow 0,
$$

where the function $f \in C^{0}(\mathbb{R}) \cap C^{2}(\mathbb{R} /\{0\})$ will be assumed throughout to satisfy the following assumptions:

(0.3) There is a real $a \leqq 0$ such that $f$ is negative and increasing on $(-\infty, a)$, with $\lim _{u \rightarrow-\infty} f(u)=-\infty$.

$$
\lim _{u \rightarrow-\infty} \frac{d f}{d u}(u) / f^{2}(u)=0 .
$$

For radial solutions $r=\|x\| \rightarrow u(r)$, the equation in $\mathbb{R}^{N} /\{0\}$ reduces to an ordinary differential equation in $(0,+\infty)$ :

$$
r^{1-N}\left(r^{N-1} u^{\prime} / \sqrt{1+u^{\prime 2}}\right)^{\prime}(r)+f(u(r))=0,
$$


where $u^{\prime}=\frac{d u}{d r}$; obviously equivalent to

$$
\frac{u^{\prime \prime}}{\left(1+u^{\prime 2}\right)^{3 / 2}}(r)+\frac{N-1}{r} \frac{u^{\prime}}{\sqrt{1+u^{\prime 2}}}(r)+f(u(r))=0 .
$$

The linear case $f(u)=(N-1) u$ is well-known problem of the pendent liquid drop. Using fixed point methods, Concus and Finn proved in [CF1] the local existence of a singular solution $U$ under the form

$$
U(r)=-\frac{1}{r}+\frac{N+3}{2(N-1)} r^{3}+r^{3} \circ(r) .
$$

Recall that $U$ has an asymptotic expansion in powers of $r$ but the formal Taylor series is divergent.

Using the same way we prove in Appendix A the local existence of a singular solution $u$ of $(0.6)$ when $f$ is a power:

$$
f(u)=\lambda|u|^{q-1} u, \quad \text { with } q, \lambda>0
$$

these rather tedious calculus lead to a solution $U$ given by

$$
U(r)=-\left(\frac{N-1}{\lambda r}\right)^{1 / q}+c_{\lambda} r^{2+1 / q}(1+\circ(r))
$$

where $c_{\lambda}=(\lambda /(N-1))^{1 / q}(q(N+1)+2) / 2(N-1)$. Notice that $r|f(U(r))|$ converges to $(N-1)$ when $r$ goes to 0 , which could be foreseen from $(0.6)$.

Another way for finding singular solutions is to consider the regular problem: let $\left(u_{0, n}\right)_{n \in \mathrm{N}}$ be any sequence of reals smaller than $a$, with $\lim _{n \rightarrow+\infty} u_{0, n}$ $=-\infty$; if $u_{n}$ is a solution of $(0.6)$ near the origin such that

$$
u_{n}(0)=u_{0, n}, \quad u_{n}^{\prime}(0)=0,
$$

one has to find the limit behaviour of $\left(u_{n}\right)_{n \in \mathbb{N}}$. This method was first investigated in the linear case when $N=2$ by Concus and Finn [CF3] and more recently by Finn [F5]. The main difficulty is that the size of the existence domain of $u_{n}$ shrinks to 0 as $n$ goes to infinity, because of the occurence of vertical points near the origin. In fact from the geometrical point of view, we can extend the notion of (regular or singular) solution of equation (0.6): we ask more generally for a $C^{2}$-embedded hypersurface in $\mathbb{R}^{N} \times \mathbb{R}$, rotationally symmetric, whose mean curvature at each point $(x, u)$ is given by $-f(u) / N$. As in [CF3] we are led to the parametrical system

$$
\left\{\begin{array}{l}
\frac{d \psi}{d s}=-f(u)-(N-1) r^{-1} \sin \psi \\
\frac{d u}{d s}=\sin \psi \\
\frac{d r}{d s}=\cos \psi
\end{array}\right.
$$


where $s$ is the arc-length of a vertical section of the hypersurface, and $\psi$ is the angle between a tangent to the section and the $r$ axis. When $\psi \in(0, \pi)$ the hypersurface can be represented by a function $r$ of $u$, and (0.11) is equivalent to

$$
\frac{\ddot{r}(u)}{\left(1+\dot{r}^{2}(u)\right)^{3 / 2}}-\frac{N-1}{r(u)} \frac{1}{\sqrt{1+\dot{r}^{2}(u)}}-f(u)=0,
$$

where $\dot{r}=\frac{d r}{d u}$, see [CF3], [APS]. The regular solutions are now represented by the solutions $r(u)$ of $(0.12)$ on $\left(u_{0, n}, a\right)$ such that

$$
\lim _{u \rightarrow u_{0, n}} r(u)=0, \quad \lim _{u \rightarrow u_{0, n}} \dot{r}(u)=+\infty .
$$

When $N=2$ and $f(u)=u$, Concus and Finn proved in [CF3] that some sequences of regular solutions converge uniformly on any compact of $\mathbb{R}^{-}$to a singular solution of $(0.12)$. They used very accurate local comparison methods with Delaunay surfaces, also called unduloids, which are rotationally symmetric surfaces with constant mean curvature. A shorter proof in [F5] shows that moreover the singular solution is locally a function $u$ of $r$; and it is asymptotic to the curve $u=-1 / r$, see Fig. 3 .

In Section 1 we give the essential tools for our study, which are energy functions of the problem. One of them is the energy function for the equation satisfied by $u^{\prime} / \sqrt{1+u^{\prime 2}}$, used in the linear case in [B2] and also in [W2]. Two other energy functions are of Pohožaev type. In fact they can be defined in the nonradial case. By integration they lead to Pohožaev relations, extending the Green's identity given in the linear case and dimension 2 in [F5].

In Section 2, our main result concerns the existence of singular solutions for a large class of functions $f$. We prove the following:

When $u \mapsto|u|^{-1 /(N-1)} f(u)$ is nondecreasing for large $|u|$, there exists a singular local generalized solution of $(0.1),(0.2)$ under the form $u \rightarrow r(u)$.

To prove this result we use one of the Pohožaev functions and some properties of unduloids in dimension $N$; we study those hypersurfaces in Appendix $\mathrm{B}$, extending some results of $[\mathbf{H Y}]$.

In Section 3 we study the local behaviour of any singular local solution of the form $u \rightarrow r(u)$. In the physical case $N=2$ we prove (under suitable assumptions on $f$ ) that $r|f(u)|$ converges to 1 when $u$ goes to $-\infty$. Our proof differs from the proof of [F5] when $f(u)=u$, since it does not use unduloids. In the case (0.8) of a power, we give also some estimates on the angle $\psi$ and the difference $|f(u)|-1 / r$. They allow us to prove that, if $q \leq 1$, 
any singular solution is a function $r \rightarrow u(r)$ (as long as $u<0$ ), which means that it has no vertical point. In dimension $N$, we extend a part of those results, generalizing the estimates of [CF2], [B2] and simplifying the first proofs.

In Section 4 we suppose that $f$ is increasing from $\mathbb{R}$ to $\mathbb{R}$ with $f(0)=0$, and give some global properties of the regular solutions, such as: global existence, estimate on the incidence angle if the curve crosses the axis $u=0$, estimates on the maximal diameter of the regular drops. This extends among others the previous results of $[\mathbf{C F 3}],[\mathbf{B 2}],[\mathbf{F 3}],[\mathbf{F 6}]$.

Acknowledgement. I am very grateful to S. SALAMEH for his contribution in numerical studies and drawings.

\section{Energy functions for regular or singular solutions.}

We call regular solution of the parametrical system (0.11) any solution such that $r>0$ for small $s>0$, with initial conditions

$$
\lim _{s \rightarrow 0} \psi(s)=0, \quad \lim _{s \rightarrow 0} u(s)=u_{0}<a, \quad \lim _{s \rightarrow 0} r(s)=0 .
$$

From [CF3], [APS] we have local existence and uniqueness of such a solution expressed in terms of $r \rightarrow u(r)$. Its satisfies locally $\sin \psi>0$, hence it is also given by a function $u \rightarrow r(u)$.

Now we call singular solution of (0.11) any solution such that $r>0, \psi \in$ $(0, \pi)$ for small $s>0$, with initial conditions

$$
\lim _{s \rightarrow 0} u(s)=-\infty, \quad \lim _{s \rightarrow 0} r(s)=0 ;
$$

in other words we look for a local positive solution $u \rightarrow r(u)$ of $(0.12)$ such that $\lim _{u \rightarrow-\infty} r(u)=0$.

Several questions follow naturally: does there exist such a solution? What is its maximal extension? Do we have $\lim _{s \rightarrow 0} \psi(s)=\frac{\pi}{2}$ ? Does the solution define a function $r \rightarrow u(r)$ ?

To deal about it, we shall use energy functions linked to the system. Some of them, used for the regular problem, are classical, see [CF3] in the linear case, and [APS] in the general case. The first one comes from integration of $\frac{d \psi}{d s} \sin \psi:$ Set

$$
E(s)=F(u)-\cos \psi,
$$

where $F(u)=\int_{a}^{u} f(t) d t$; then $E$ is nonincreasing, since

$$
\frac{d E}{d s}(s)=-(N-1) r^{-1} \sin ^{2} \psi .
$$


The second one comes from integration of $r^{N-1} \frac{d \psi}{d s} \cos \psi$ : let us define

$$
J(s)=r^{N-1} \sin \psi+r^{N} f(u) / N,
$$

then

$$
\frac{d J}{d s}(s)=\frac{r^{N}}{N} \dot{f}(u) \sin \psi
$$

therefore $J$ is increasing as long as $\psi \in(0, \pi)$. In fact it is also positive, which gives an essential estimate for singular solutions as well as for regular ones:

Proposition 1.1. Any singular (resp. regular) solution $r(u)$ is defined at least on $(-\infty, a)$ (resp. $\left.\left(u_{0}, a\right)\right)$ and satisfies the relation

$$
0<r|f(u)|<N \sin \psi
$$

Proof. Let us recall the proof of [APS] in the regular case and extend it to the singular one. Near the origin, $J$ is increasing; let $\ell=\lim _{s \rightarrow 0} J(s) \in$ $[-\infty,+\infty)$. Obviously $\ell=0$ in the regular case. In the singular case $\ell$ is nonpositive, since $f(u) \leqq 0$. Suppose $\ell<0$; then $\lim _{u \rightarrow-\infty}(\cos \psi)(u) / f(u)=$ 1 , since $(\cos \psi)^{\circ}=(N-1) r^{-1} \sin \psi+f(u)$; and $\cos \psi-F(u) / 2$ is decreasing for large $|u|$, which is impossible. Then $\ell=0$ and $J(s)$ is positive near 0 , which means that $(1.7)$ is satisfied near 0 . Let $(-\infty, \bar{a})\left(\operatorname{resp} .\left[-u_{0}, \bar{a}\right)\right)$ its maximal existence set. If $\bar{a}<a$, then $r$ and $\frac{d J}{d s}$ cannot vanish, from (1.5), (1.6); hence $\liminf _{r \rightarrow \bar{a}} r(u)>0$, and $|\dot{r}|$ is bounded by $N / r|f(u)|$; then $\bar{a}$ is not maximal. Hence $\bar{a} \geqq a$ and (1.7) is true up to $a$.

The function $E$ gives also useful properties of the vertical points. The proofs of [APS] are similar in the singular case:

Proposition 1.2. The extremal points of any singular (resp. regular) solution $r(u)$ are isolated in $(-\infty, a]\left(\operatorname{resp}\left[u_{0}, a\right]\right)$. There is an $\bar{u}_{0}<0$ such that any regular solution has more than one extremal point when $u_{0}<\bar{u}_{0}$, one exactly when $u=\bar{u}_{0}$ and no extremal point when $u_{0}>\bar{u}_{0}$, if $a>\bar{u}_{0}$. Denoting by $\left(u_{n}\right)$ the decreasing sequence of the extremal points, finite or infinite, we have

$$
r\left(u_{2 n}\right)\left|f\left(u_{2 n}\right)\right|>N-1>r\left(u_{2 n+1}\right)\left|f\left(u_{2 n+1}\right)\right|,
$$


for any maximal point $u_{2 n}(n \geqq 0$ or 1$)$ and any minimal point $u_{2 n+1}(n \geqq 0)$. And the sequences $\left(r\left(u_{2 n}\right)\right)$ and $\left(r\left(u_{2 n+1}\right)\right)$ are decreasing with $n$.

Remark 1.1. Between two consecutive extremal points the curve crosses the "basic curve" $r=-(N-1) / f(u)$ exactly one time. It has no inflection point on the set of $u$ where $r|f(u)|>N-1$, from (1.9).

The third energy function was used in the linear case in [B2] to prove the global existence of singular solutions, and in [W2] to study the stability of the regular viles. It is new in the nonlinear case. In fact it is the energy function for the equation obtained by derivation of (0.6) by respect to $r$ (or (0.12) by respect to $u$ ); let us denote

$$
\xi=\cos \psi=\dot{r} / \sqrt{1+\dot{r}^{2}}
$$

then (0.11), (0.12) imply

$$
r \dot{\xi}=r \ddot{r}\left(1+\dot{r}^{2}\right)^{3 / 2}=-r \frac{d \psi}{d s}=r f(u)+(N-1) \sqrt{1-\xi^{2}} .
$$

Let us define, for any $u<a$, the function

$$
G(u)=\frac{r^{2}}{2} \dot{\xi}^{2}+\frac{N-1}{2} \xi^{2}-r^{2} \dot{f}(u) \xi
$$

then

$$
\dot{G}(u)=-(N-2) r \frac{\xi \dot{\xi}^{2}}{\sqrt{1-\xi^{2}}}-2 r \dot{f}(u) \frac{\xi^{2}}{\sqrt{1-\xi^{2}}}-r^{2} \ddot{f}(u) \xi .
$$

Notice that $G$ is nonincreasing when $\xi \geqq 0$ and $\ddot{f}(u) \geqq 0$.

The last functions we shall use in our study are Pohožaev type functions: for any $q>0$ and $\delta \geqq 0$ we define, for any $u<a$,

$$
\phi_{\delta, q}(u)=r^{N}\left(\delta-\cos \psi+\frac{u f(u)}{q+1}+\frac{N}{q+1} \frac{u \sin \psi}{r}\right) ;
$$

then

$$
\begin{aligned}
\dot{\phi}_{\delta, q}(u)=-\frac{r^{N-1}}{(q+1) \sin \psi} & \left((N+q+1) \cos ^{2} \psi-N \delta(q+1) \cos \psi\right. \\
+ & (N-1) q-1+(q f(u)-u \dot{f}(u)) r \sin \psi) ;
\end{aligned}
$$

then $\phi_{\delta, q}$ is nonincreasing when $q \geqq 1 /(N-1), u \rightarrow|u|^{-q} f(u)$ is nondecreasing, and $0 \leqq \delta \leqq \delta_{q}$, where

$$
\delta_{q}=\left\{\begin{array}{l}
1, \quad \text { if } q \geqq(N+2) /(N-2) \\
\frac{2}{N(q+1)}((N+q+1)((N-1) q-1))^{1 / 2}<1, \quad \text { if not. }
\end{array}\right.
$$


This function is very close to the function

$$
\psi_{\delta, q}(u)=r^{N}\left(\delta-\cos \psi+F(u)+\frac{N}{q+1} u \frac{\sin \psi}{r}\right)
$$

which gives by differentiation,

$$
\begin{aligned}
\dot{\psi}_{\delta, q}(u)=-\frac{r^{N-1}}{(q+1) \sin \psi}\left((N+q+1) \cos ^{2} \psi-N \delta(q+1) \cos \psi\right. \\
+(N-1) q-1+N \cos \psi(u f(u)-(q+1) F(u)))
\end{aligned}
$$

The function $\psi_{1,(N+2) /(N-2)}$ was used for the study of ground states $r \rightarrow u(r)$ of $(0.6)$ in [NiS1]; and $\phi_{1,(N+2) /(N-2)}$ for ground states $u \rightarrow r(u)$ of $(0.12)$ in [APS], because $\cos \psi$ does not keep a constant sign.

Remark 1.2. Consider more generally the nonradial case of an orientable $C^{2}$ hypersurface $S$ in $\mathbb{R}^{N+1}$, given by $X\left(x_{1}, \ldots, x_{N}, x_{N+1}\right)$, governed by the equation

$$
\Delta_{S} X=f\left(x_{N+1}\right) \bar{n}
$$

where $\bar{n}$ is the unit exterior normal to $S$, and $\Delta_{S}$ is the Laplace-Beltrami operator. Suppose that for any $u$ in an interval $I \subset(-\infty, a)$ the hyperplane $H_{u}: x_{N+1}=u$ cuts $S$ in an hypersurface $\Sigma_{u}$ of $\mathbb{R}^{N-1}$, enclosing a domain $\Omega_{u}$ of $\mathbb{R}^{N}$, regular enough. Let $n$ be the unit exterior normal to $\Sigma_{u}$ in $H_{u}$, and $\psi$ be the angle between $S$ and $H_{u}$ on $\Sigma_{u}$. For any $q>0$ and $\delta \geqq 0$, let us define the function

$$
\bar{\Phi}_{\delta, q}(u)=\int_{\Sigma_{u}}\left(\left(\delta-\cos \psi+\frac{u f(u)}{q+1}\right) X . n+N \frac{u \sin \psi}{q+1}\right) d \Sigma_{u} .
$$

Then we prove in [B3] that, for any $u \in I$,

$$
\begin{aligned}
\dot{\bar{\phi}}_{\delta, q}(u)= & -\frac{1}{(q+1)} \\
& \cdot \int_{\Sigma_{u}}\left(\frac{1}{\sin \psi}\left((N+q+1) \cos ^{2} \psi-N(q+1) \delta \cos \psi+(N-1) q-1\right)\right. \\
& +(q f(u)-u \dot{f}(u)) X . n) d \Sigma_{u} ;
\end{aligned}
$$

hence $\bar{\Phi}_{\delta, q}$ is nondecreasing when $q \geqq 1 /(N-1), u \rightarrow|u|^{-q} f(u)$ is nondecreasing and $\delta \leqq \delta_{q}$. 
In particular if $S$ has a finite minimum $u_{0} \in I$, we get by integration between $u_{0}$ and $u \in I$ the following Pohožaev relation:

$$
\begin{aligned}
& ((q+1) \delta+u f(u))\left|\Omega_{u}\right|-\frac{q+1}{N} \int_{\Sigma_{u}} X \cdot n \cos \psi d \Sigma_{u}+u \int_{\Sigma_{u}} \sin \psi d \Sigma_{u} \\
& =-\frac{1}{N} \int_{S_{u}}\left((N+q+1) \cos ^{2} \psi-N(q+1) \delta \cos \psi+(N-1) q-1\right) d S_{u} \\
& \quad-\int_{V_{u}}(q f(u)-u \dot{f}(u)) d V_{u},
\end{aligned}
$$

where $S_{u}$ is the part of $S$ under $H_{u}$, and $V_{u}$ is the domain of $\mathbb{R}^{N+1}$ with boundary $S_{u} \cup \Omega_{u}$.

When $\delta=0, N=2$ and $f(u)=u$ we find again the Green's identity of Finn, see [F5].

Remark 1.3. It is interessant to notice that, when (and only when) $N=2$ and $f(u)=u$, then, up to a constant, the function $G$ coincides with the Pohožaev function $\phi_{0,1}$ :

$$
G(u)=\phi_{0,1}(u)+\frac{1}{2}=\frac{1}{2}(r u+\sin \psi)^{2}+\frac{\cos ^{2} \psi}{2}-r^{2} \cos \psi
$$

Hence in this case the use of $G$, or $\phi_{0,1}$, is equivalent to the use of Green's identity of [F5].

Remark 1.4. Let us mention at least an energy function used in [FLS]: it is

$$
\Gamma(s)=r^{2(N-1)}\left(\sin ^{2} \psi+2 F(u) \cos \psi-F^{2}(u)\right),
$$

which is nondecreasing when $F(u) \leqq 2$, since

$$
\frac{d \Gamma}{d s}(s)=2(N-1) r^{2 N-3} F(u)\left(\cos ^{2} \psi-F(u) \cos \psi+1\right) .
$$

\section{Existence results.}

First we consider the case where $f$ is a power, given by (0.8), where $\lambda$ is a positive parameter. The change of variables $u(r)=a^{-1} v(a r)$, where $a=(\lambda /(N-1))^{1 /(q-1)}$ reduces the problem to the case where $\lambda=N-1$. Using the fixed point method of [CF2] we get the following, see Appendix A:

Theorem 2.1. For any $q>0$, there exists a local solution $r \rightarrow U(r)$ of the equation

$$
\frac{u^{\prime \prime}}{\left(1+u^{\prime 2}\right)^{3 / 2}}(r)+\frac{N-1}{r} \frac{u^{\prime}}{\sqrt{1+u^{\prime 2}}}(r)+(N-1)|u(r)|^{q-1} u(r)=0
$$


such that

$$
U(r)=-r^{-1 / q}+\frac{q(N+1)+2}{2(N-1)} r^{2+1 / q}(1+\circ(r)) .
$$

Remark 2.1. As in [CF2] one can obtain an asymptotic expansion for $U(r)$ in powers of $r$ near 0 , which only contains powers of the form $r^{2+1 / q+2 p(1+1 / q)}(p \in \mathbb{N})$. Notice that $U$ is asymptotic to the curve $r \rightarrow-r^{-1 / q}$ and more precisely $1 / r+f(U) /(N-1)=1 / r-|U|^{q}=0\left(r^{1+2 / q}\right)$. A last $U$ is locally concave. The question of uniqueness is open. In [B2] we proved the uniqueness among locally concave functions when $q=1$.

The fixed point method gives accurate results but we could not extend it to more general functions $f$. Now, following the idea of [F5] we start from the regular solutions.

\section{Theorem 2.2. Suppose that}

$$
u \rightarrow|u|^{-1 /(N-1)} f(u) \text { is nondecreasing on }(-\infty, a) \text {; }
$$

then there exist $b<a$ and a sequence of regular solutions of (0.12) converging uniformly on any compact set of $(-\infty, b]$ to a singular solution of the equation.

Proof. For any $u_{0}<a$, denote by $r\left(., u_{0}\right)$ the regular solution starting from $u_{0}$. Let $b<a-1$ such that $|f(b+1)|>1 / 2 \sqrt{2}$, and let $d<b$. We are going to prove as in $[\mathbf{F 5}]$ that

(2.4) There exist $\varepsilon>0$ and $A<d$ such that $\inf _{[d, b] \times(-\infty, A]} r\left(u, u_{0}\right) \geqq \varepsilon$.

Suppose it is false. Then for any sequence $\varepsilon_{n} \rightarrow 0$ there are $\widetilde{u}_{n} \in[d, b], u_{0, n} \rightarrow$ $-\infty$, such that $\widetilde{r}_{n}=r\left(\widetilde{u}_{n}, u_{0, n}\right)=\min _{[d, b]} r\left(u, u_{0, n}\right) \leqq \varepsilon_{n}$. From (0.4)we can take $d$ large enough such that $\dot{f}(u) / f^{2}(u)<1 /(1+\sqrt{2})$ on $(-\infty, d)$ and $|f(d)|>2$, and take $\varepsilon_{0}<\min (1 / 2,(N-1) / 3 \sqrt{2}|f(d-1)|)$. Then we claim that

There is a $u_{n} \in[d-1, b+1]$ such that $r_{n}=r\left(u_{n}, u_{0, n}\right) \leqq \varepsilon_{n}$ and $r_{n}$ is a minimal point of the curve $r\left(., u_{0, n}\right)$.

Indeed either $\widetilde{r}_{n}$ is a minimal point, or $\widetilde{u}_{n}=b$ and $\dot{r}\left(b, u_{0, n}\right)<0$, or $\widetilde{u}_{n}=d$ and $\dot{r}\left(d, u_{0, n}\right)>0$. When $\widetilde{u}_{n}=b$ (resp. $\widetilde{u}_{n}=d$ ) there exists a $u_{n} \in\left[b, b+\varepsilon_{n}\right]$ with $r_{n}=r\left(u_{n}, u_{0, n}\right)<\widetilde{r}_{n}$ and $\dot{r}_{n}=\dot{r}\left(u_{n}, u_{0, n}\right) \in(-1,0]$ (resp. $u_{n} \in$ 
$\left[d, d-\varepsilon_{n}\right]$ with $r_{n}<\widetilde{r}_{n}$ and $\left.\dot{r}_{n} \in[0,1)\right)$, since $r$ remains positive. When $\widetilde{u}_{n}=b$, we get $\ddot{r}_{n} /\left(1+\dot{r}_{n}^{2}\right)^{3 / 2} \geqq 2\left|f\left(u_{n}\right)\right|>0$, from (0.12). Then for $u-u_{n}>0$ small enough, we get again $\ddot{r} /\left(1+\dot{r}^{2}\right)^{3 / 2} \geqq 2\left|f\left(u_{n}\right)\right|$, and this is true as long as $\dot{r}$ is negative, since $|f(u)|<\left|f\left(u_{n}\right)\right|$ and $\dot{r}^{2}$ is decreasing. By integration we deduce that $\dot{r} / \sqrt{1+\dot{r}^{2}}+1 / \sqrt{2} \geqq 2|f(b+1)|\left(u-u_{n}\right)$, hence there is a minimal point in $[b, b+1]$. When $\widetilde{u}_{n}=d$ we get again $\ddot{r}_{n}>0$, and for $u-u_{n}<0$ small enough we have $\ddot{r} /\left(1+\dot{r}^{2}\right)^{3 / 2} \geqq 3\left|f\left(u_{n}\right)\right|-|f(u)|$; and this is true as long as $\dot{r}$ is positive and $|f(u)| \leqq 3\left|f\left(u_{n}\right)\right|$. By integration we deduce that

$$
\begin{aligned}
1 / \sqrt{2}-\dot{r} / \sqrt{1+\dot{r}^{2}} & \geqq F\left(u_{n}\right)-F(u)+3\left|f\left(u_{n}\right)\right|\left(u_{n}-u\right) \\
& \geqq\left(3\left|f\left(u_{n}\right)\right|-|f(u)|\right)\left(u_{n}-u\right) .
\end{aligned}
$$

Then there is a minimal point in $[d-1, d]$ : if not, taking $u=u_{n}-1 /\left|f\left(u_{n}\right)\right|$ in (2.6), we would have $1 / \sqrt{2} \geqq 3-|f(u)| /\left|f\left(u_{n}\right)\right|$; but $1 /|f(u)|=1 /\left|f\left(u_{n}\right)\right|(1-$ $\left.\left(\dot{f} / f^{2}\right)\left(\xi_{n}\right)\right)$ with $\xi_{n} \in\left(u, u_{n}\right)$, hence $1 / \sqrt{2} \geqq 2-1 / \sqrt{2}$, which is false. Then $(2.5)$ is proved.

Now we use for $r\left(., u_{0, n}\right)$ the Pohožaev function $\phi=\phi_{0,1 /(N-1)}$ defined in (1.12) by

$$
\phi(u)=r^{N}\left(-\cos \psi+\frac{N-1}{N} u f(u)+(N-1) u \frac{\sin \psi}{r}\right)
$$

from (2.3) $\phi$ is decreasing with $u$ and

$$
\phi(u)=-r^{N-1}\left(N \frac{\cos ^{2} \psi}{\sin \psi}+\frac{r}{N}\left(\frac{f(u)}{N-1}-u \dot{f}(u)\right)\right) .
$$

By integration we get

$$
N \int_{u_{0, n}}^{u_{n}} r^{N-1} \frac{\cos ^{2} \psi}{\sin \psi} d u \leqq\left|\phi\left(u_{n}\right)\right|=0\left(\varepsilon_{n}^{N-1}\right)
$$

since $u_{n}$ remains in $[d-1, b+1]$. On the other hand we can also choose $\varepsilon_{0}$ such that $\varepsilon_{0}<(N-1) /|f(d-1-N M \sqrt{2})|$, where $M$ is defined in App. B, Lemma B.2. From Lemma B.2, there is an unduloid $\Omega_{k_{n}}$, with extremal points $\left(u_{n}, r_{n}\right),\left(v_{n}, \sigma_{n}\right)$, with $v_{n}<u_{n}, \sigma_{n}>r_{n}$ and curvature $\left|f\left(v_{n}\right)\right| / N$. As in [F5] it lies below the curve $r\left(., u_{0, n}\right)$ with an angle $\theta \in\left(\frac{\pi}{2}, \psi\right)$ for any $r \in\left(r_{n}, \sigma_{n}\right)$; then

$$
\begin{aligned}
\left|\phi\left(u_{n}\right)\right| / N \geqq \int_{v_{n}}^{u_{n}} r^{N-1} \frac{\cos ^{2} \psi}{\sin \psi} d u & \geqq \int_{r_{n}}^{\sigma_{n}} r^{N-1}|\cos \psi| d r \\
& \geqq \int_{r_{n}}^{\sigma_{n}} r^{N-1}|\cos \theta| d r
\end{aligned}
$$


But the last integral is bounded below by a positive constant; indeed from Lemma B.1, the sequence $\left(v_{n}\right)$ is bounded, hence we can extract a subsequence $\left(v_{\nu}\right)$ of limit $v$. From Lemma B.2, we have $\lim k_{\nu}=1, \lim \sigma_{\nu}=$ $N /|f(v)|>0$; then the curves $\Omega_{k_{\nu}}$ tend to a lower quarter circle with center $(0, v)$ and radius $N /|f(v)|$. Hence we get $(2.4)$ by contradiction.

Now from (1.7) we have, for any $\left(u, u_{0}\right) \in[d, b] \times(-\infty, A]$,

$$
\sqrt{1+\dot{r}^{2}}<N / r|f(u)|<N / \varepsilon|f(b)|
$$

hence

$$
\sup _{[d, b] \times(-\infty, A]}\left|\dot{r}\left(u, u_{0}\right)\right|<+\infty .
$$

From equation (0.12) it is the same for the higher derivatives. Then for any sequence of regular solutions of $(0.12)$, with initial data $u_{0, n} \rightarrow-\infty$, there is a subsequence converging uniformly on any compact set of $(-\infty, b]$ to a positive function $u \rightarrow r(u)$, solution of $(0.12)$ on $(-\infty, b]$. Moreover, going to the limit in (1.7) for regular solutions, we get $0<r(u)|f(u)| \leqq N \sin \psi$, hence $\lim _{u \rightarrow-\infty} r(u)=0$ and $u \rightarrow r(u)$ is singular solution of (0.11). From Proposition 1.1 , it can be extended to $(-\infty, a)$ and satisfies $(1.7)$ on the whole interval.

Remark 2.2. The assumption (2.3) is linked to the Pohožaev function $\phi$; it means that $f$ does not increase too slowly at infinity. It is satisfied in particular by any function $f$ such that $x \mapsto|f(-x)|$ is convex for large $x>0$. When $f(u)=|u|^{q-1} u$, it reduces to the condition $q \geqq 1 /(N-1)$. Comparing with the result of Theorem 2.1, we conjecture that (2.3) is not needed.

Remark 2.3. The method of comparison with an unduloid such $\Omega_{k, n}$, defined implicitely, was used in $[\mathbf{F 5}]$ and also in $[\mathbf{I}]$ to study the bounds of the radius $r(0)$ when $N=2$ and $f(u)=|u|^{q-1} u$ with $q>1$.

\section{Local behaviour of the singular solutions.}

Here we look at the behaviour near $-\infty$ of any singular solution $u \rightarrow r(u)$. Either it is decreasing near $-\infty$, hence it defines a function $r \rightarrow u(r)$. Or the sequence of extremal points is infinite, and $r(u)$ crosses the basic curve $r=-(N-1) / f(u)$ infinitely many times.

Our best results concern the case $N=2$. At first, under simple assumptions on $f$, we prove that the curve is asymptotic to the basis curve:

Theorem 3.1. Suppose that $N=2$, and $f$ satisfies

$$
\ddot{f} / f^{2} \in L^{1}(-\infty, a) \quad \text { and } \quad \lim _{u \rightarrow-\infty}(\ddot{f} / f \dot{f})(u)=0 .
$$


Then any singular solution satisfies

$$
\begin{aligned}
& \lim _{u \rightarrow-\infty} r(u) f(u)=-1, \\
& \lim _{u \rightarrow-\infty} \dot{r}(u)=0 \quad\left(\text { that is } \lim _{u \rightarrow-\infty} \psi(u)=\frac{\pi}{2}\right) .
\end{aligned}
$$

Proof. Here we use the energy function defined in (1.10) for any singular solution $r(u)$ : let

$$
G(u)=r^{2} \frac{\dot{\xi}^{2}}{2}+\frac{\xi^{2}}{2}-r^{2} \dot{f}(u) \xi
$$

where $\xi=\cos \psi ;$ then, since $N=2$,

$$
\dot{G}(u)=-2 r \dot{f}(u) \frac{\xi^{2}}{\sqrt{1-\xi^{2}}}-r^{2} \ddot{f}(u) \xi
$$

From Proposition 1.1 and assumption (3.1) the function $u \rightarrow r^{2} \ddot{f}(u) \xi$ is integrable on $(-\infty, a)$. Set

$$
W(u)=\int_{-\infty}^{u} r^{2} \ddot{f}(w) \xi d w ;
$$

then the function $H=G+W$ is nonincreasing; it is bounded near $-\infty$; indeed from (1.7), (1.9) we have

$$
|r \dot{\xi}(u)|=\left|r f(u)+\sqrt{1-\xi^{2}}\right| \leqq 3 ;
$$

moreover $\left|r^{2} \dot{f}(u)\right| \leqq 2 \dot{f}(u) / f^{2}(u)$, hence from (0.4)

$$
\lim _{u \rightarrow-\infty} r^{2} \dot{f}(u)=0 .
$$

Then $H$ has a finite limit $\ell / 2$ at $-\infty$, and from (3.6), (3.8), we get

$$
\lim _{u \rightarrow-\infty}\left(r^{2} \dot{\xi}^{2}+\xi^{2}\right)=\ell \geqq 0 .
$$

Suppose first that $\ell$ is positive. Let $b<a$ and

$$
v(u)=\int_{b}^{u} \frac{d w}{r(w)}<0 \text { for any } u<b ;
$$

then, from (1.7), $N|v(u)| \geqq F(u)-F(b)$, and $\lim _{u \rightarrow-\infty} v(u)=-\infty$. Taking $v$ as a new variable, (3.9) becomes

$$
\lim _{v \rightarrow-\infty}\left(\left(\frac{d \xi}{d v}\right)^{2}+\xi^{2}\right)=\ell
$$


If $\xi$ keeps a constant sign, then $\lim _{v \rightarrow-\infty} \xi= \pm \sqrt{\ell}$ and $\lim _{v \rightarrow-\infty} \frac{d \xi}{d v}=0$; since $\dot{r}=\xi / \sqrt{1-\xi^{2}}$ then there is a $c>0$ such that $|\dot{r}(u)|>c$ for large $|u|$; this is impossible since $\lim _{u \rightarrow-\infty} r=0$. Then $\xi$ is necessarily oscillating near infinity and it least period in $v$ goes to $2 \pi$. Let $\left(u_{n}\right)$ be the decreasing sequence of the zeros of $\xi$ less than $b$ : we can suppose that $r_{2 n}=r\left(u_{2 n}\right)$ is a maximal point, hence $r_{2 n}\left|f\left(u_{2 n}\right)\right|>1$ and $r_{2 n+1}$ is a minimal point with $r_{2 n+1}\left|f\left(u_{2 n+1}\right)\right|<1$.

From (1.7), (3.10) we have

$$
v\left(u_{n}\right)-v\left(u_{n+1}\right)=\int_{u_{n+1}}^{u_{n}} \frac{d w}{r(w)} \geqq \frac{1}{2} \int_{u_{n+1}}^{u_{n}}|f(w)| d w \geqq \frac{1}{2}\left|f\left(u_{n}\right)\right|\left(u_{n}-u_{n+1}\right) ;
$$

this implies the estimate

$$
u_{n}-u_{n+1} \leqq \frac{5 \pi}{\left|f\left(u_{n}\right)\right|} \quad \text { for large } n .
$$

Since $1 / f\left(u_{n+1}\right)-1 / f\left(u_{n}\right)=\left(u_{n+1}-u_{n}\right) \dot{f}\left(\lambda_{n}\right) / f^{2}\left(\lambda_{n}\right)$ for some $\lambda_{n} \in\left[u_{n+1}, u_{n}\right]$, we get, from (0.4) and (3.13),

$$
\lim _{r \rightarrow+\infty} f\left(u_{n+1}\right) / f\left(u_{n}\right)=1 ;
$$

hence for large $n$,

$$
\left|f\left(u_{n}\right)\right| \leqq\left|f\left(u_{n+1}\right)\right| \leqq 2\left|f\left(u_{n}\right)\right|
$$

Now the function

$$
u \rightarrow-\frac{\dot{H}(u)}{2}=r \dot{f}(u) \frac{\xi^{2}}{\sqrt{1-\xi^{2}}}
$$

is in $L^{1}(-\infty, a)$, since $H$ decreases to a finite limit. Let us look at its integral between $u_{2 n+1}$ and $u_{2 n}$; let $w_{n} \in\left(u_{2 n+1}, u_{2 n}\right)$ be the unique intersection point with the basis curve: $r\left(w_{n}\right)\left|f\left(w_{n}\right)\right|=1$. From (1.9), $\xi$ is decreasing on $\left[w_{n}, u_{2 n}\right]$, and from $(3.7),(3.9), \lim \xi^{2}\left(w_{n}\right)=\ell(1-\ell / 4) \in[3 \ell / 4,3 / 4]$. Then $\xi_{n}=\xi\left(w_{n}\right) \in(\sqrt{\ell} / 2, \sqrt{7 / 8})$ for large $n$. Now $r|f(u)| \geqq 1$ on $\left[w_{2 n}, u_{2 n}\right]$; then

$$
\begin{aligned}
\int_{u_{2 n+1}}^{u_{2 n}} r \dot{f}(u) & \frac{\xi^{2}}{\sqrt{1-\xi^{2}}} d u \geqq \int_{w_{2 n}}^{u_{2 n}} r \dot{f}(u) \frac{\xi^{2}}{\sqrt{1-\xi^{2}}} d u \\
& \geqq \int_{w_{2 n}}^{u_{2 n}} \frac{\dot{f}(u)}{|f(u)|} \frac{\xi^{2}}{\sqrt{1-\xi^{2}}} d u \geqq \int_{\xi_{n} / 2}^{\xi_{n}} \frac{\dot{f}(u)}{|f(u)|} \frac{\xi^{2}}{\sqrt{1-\xi^{2}}} \frac{d \xi}{|\dot{\xi}|} .
\end{aligned}
$$


From (3.9) we have on $\left[\xi_{n} / 2, \xi_{n}\right]$

$$
\dot{\xi}^{2} / f^{2}(u) \leqq r^{2} \dot{\xi}^{2} \leqq 17 \ell / 16-\xi^{2} \leqq \ell,
$$

for large $n$; hence there is an $\eta_{n} \in\left[u_{2 n+1}, u_{2 n}\right]$ such that

$$
\begin{aligned}
\int_{u_{2 n+1}}^{u_{2 n}} r \dot{f}(u) \frac{\xi^{2}}{\sqrt{1-\xi^{2}}} d u & \geqq \frac{1}{8} \sqrt{\frac{2}{\ell}} \int_{\xi_{n} / 2}^{\xi_{n}} \frac{\dot{f}(u)}{f^{2}(u)} d \xi \geqq \frac{\sqrt{2}}{32} \frac{\dot{f}\left(\eta_{n}\right)}{f^{2}\left(\eta_{n}\right)} \\
& \geqq \frac{\sqrt{2}}{640 \pi} \frac{\dot{f}\left(\eta_{n}\right)}{\left|f\left(\eta_{n}\right)\right|}\left(u_{2 n}-u_{2 n+2}\right)
\end{aligned}
$$

from $(3.13),(3.15)$. Let us prove that the series $\sum \frac{\dot{f}\left(\eta_{n}\right)}{\left|f\left(\eta_{n}\right)\right|}\left(u_{2 n}-u_{2 n+2}\right)$ is divergent: let $\tau_{n}$ (resp. $\sigma_{n}$ ) be a maximal (resp. minimal) point of $\dot{f}$ on $\left[u_{2 n+2}, u_{2 n}\right]$. Then there is a $\zeta_{n} \in\left[\tau_{n}, \sigma_{n}\right]$ such that

$$
\dot{f}\left(\sigma_{n}\right)-\dot{f}\left(\tau_{n}\right)=\left(\sigma_{n}-\tau_{n}\right) \ddot{f}\left(\zeta_{n}\right),
$$

then from (3.13), (3.15),

$$
\left|\dot{f}\left(\sigma_{n}\right)-\dot{f}\left(\tau_{n}\right)\right| / \dot{f}\left(\zeta_{n}\right) \leqq 10 \pi\left|(\ddot{f} / f \dot{f})\left(\zeta_{n}\right)\right| ;
$$

from (3.1) we get $\left|\dot{f}\left(\sigma_{n}\right)-\dot{f}\left(\tau_{n}\right)\right| \leqq \dot{f}\left(\zeta_{n}\right) / 2 \leqq \dot{f}\left(\tau_{n}\right) / 2$, then $\dot{f}\left(\eta_{n}\right) \geqq \dot{f}\left(\tau_{n}\right) / 2$, and

$$
\frac{\dot{f}\left(\eta_{n}\right)}{\left|f\left(\eta_{n}\right)\right|}\left(u_{2 n}-u_{2 n+2}\right) \geqq \frac{1}{4} \int_{u_{2 n+2}}^{u_{2 n}} \frac{\dot{f}(u)}{|f(u)|} d u
$$

Hence the series is divergent, since $\dot{f} /|f|$ is not integrable at $-\infty$. By contradiction we deduce that

$$
\lim _{u \rightarrow-\infty} G(u)=\lim _{u \rightarrow-\infty}\left(r^{2} \dot{\xi}^{2}+\xi^{2}\right)=\lim _{u \rightarrow-\infty}\left((r f(u)+\sin \psi)^{2}+\cos ^{2} \psi\right)=0 .
$$

Then we get (3.2) and (3.3).

Remark 3.1. Assumptions (0.4), (3.1) are satisfied in particular by $f(u)=$ $-e^{|u|^{k}}$ for any positive $k$, by the powers $f(u)=|u|^{q-1} u$ for any $q>0$, by $f(u)=-\log (1+|u|)$, etc $\ldots$; similar assumptions were introduced in [A]. When $f$ satisfies $(0.3),(0.4)$ and is convex or concave, then the assumption $\ddot{f} / f^{2} \in L^{1}(-\infty, a)$ is equivalent to: $\dot{f}^{2} / f^{3} \in L^{1}(-\infty, a)$, since

$$
\int_{-\infty}^{u}\left(\ddot{f} / f^{2}\right)(t) d t=\left(\dot{f} / f^{2}\right)(u)+2 \int_{-\infty}^{u}\left(\dot{f}^{2} / f^{3}\right)(t) d t
$$


and then $\left(\dot{f} / f^{2}\right)=\ddot{f} / f^{2}-2 \dot{f}^{2} / f^{3} \in L^{1}(-\infty, a)$.

Remark 3.2. Under the assumptions of Theorem 3.1, we have obtained (3.20). By integration we get from (3.4), (3.5) the following relation on $(-\infty, a)$ :

$$
\begin{aligned}
r^{2} \dot{\xi}^{2}+\left(\xi-r^{2} \dot{f}(u)\right)^{2}+4 \int_{-\infty}^{u} r \dot{f}(t) \frac{\xi^{2}}{\sqrt{1-\xi^{2}}} d t & =r^{4} \dot{f}^{2}(u)-2 \int_{-\infty}^{u} r^{2} \ddot{f}(t) \xi d t \\
& \leqq r^{4} \dot{f}^{2}(u)+2 \int_{-\infty}^{u} r^{2}|\ddot{f}(t)| d t .
\end{aligned}
$$

Hence with (3.2) we deduce the estimates

$$
|\xi|=|\cos \psi| \leqq\left(2\left(\dot{f} / f^{2}\right)(u)+\sqrt{2}\left(\int_{-\infty}^{u}\left(\ddot{f} / f^{2}\right)(t) d t\right)^{1 / 2}\right)(1+\circ(1)),
$$

$$
|r f(u)-1| \leqq\left(\left(\dot{f} / f^{2}\right)(u)+\sqrt{2}\left(\int_{-\infty}^{u}\left(\ddot{f} / f^{2}\right)(t) d t\right)^{1 / 2}\right)(1+\circ(1)) .
$$

When $f$ is convex for large $|u|$ (which means that $x \rightarrow|f(-x)|$ is concave for large $x>0$ ) we deduce from (3.22) that

$$
\max (|\xi|,|r f(u)-1|) \leqq \sqrt{2}\left(\dot{f} / f^{2}\right)(u)(1+\circ(1)) .
$$

When $f$ is a power we can give more accurate estimates and prove that, in case of convexity, the singular curves $u \rightarrow r(u)$ cannot admit a vertical point on $(-\infty, a)$; in the linear case we find again the result of $[\mathbf{F 5}]$ by another method.

Theorem 3.2. Suppose $N=2$ and $f(u)=|u|^{q-1} u$ for any real $u$. Then:

(i) If $0<q \leqq 1$, any singular curve can be expressed as a function $r \rightarrow u(r)$ as long as $u \leqq 0$.

(ii) For any $q>0$, the singular curves $u \rightarrow r(u)$ satisfies the following estimates near $-\infty$, with $c=|q-1| \sqrt{q / 8(q+1)}$ :

$$
\begin{gathered}
-\frac{c}{|u|^{q+1}}(1+\circ(1)) \leqq \cos \psi \leqq \frac{2 q+c}{|u|^{q+1}}(1+\circ(1)), \\
\left.|| u\right|^{q}-1 / r \mid \leqq(q+c) r^{1 / q}(1+\circ(1)) .
\end{gathered}
$$

Proof. When $q=1$, then $W=0, G$ is nonincreasing from 0 , hence nonpositive. From (3.4) we have $\xi(u) \geqq 0$ for any $u \in(-\infty, a)$. If $\xi(\bar{u})=0$ 
for some $\bar{u}<0$, then $\dot{\xi}(\bar{u})=0, G(\bar{u})=0$ and $G \equiv 0$ on $(-\infty, \bar{u})$; that means that $\dot{r} \equiv 0$ and $r f(u)+1 \equiv 0$ on $(-\infty, \bar{u})$, which is impossible. Hence $\xi(u)=\dot{r}(u)>0$ and the curve can be represented as a function $r \rightarrow u(r)$ as long as $u<0$.

Now consider any $q>0$. Let $\varepsilon>0$; from (3.2), (3.22) we have for large $|u|$

$$
\begin{aligned}
r^{2} \dot{\xi}^{2}+\left(\xi-q r^{2}|u|^{q-1}\right. & )^{2}+\frac{4 q}{1+\varepsilon} \int_{-\infty}^{u} \frac{\xi^{2}}{|t|} d t \\
& \leqq\left(q r^{2}|u|^{q-1}\right)^{2}+2 q|q-1|(1+\varepsilon) \int_{-\infty}^{u} \frac{|\xi|}{|t|^{q+2}} d t
\end{aligned}
$$

From Hölder inequality we deduce that, for any $\alpha>0$,

$$
\begin{aligned}
r^{2} \dot{\xi}^{2}+\left(\xi-q r^{2}|u|^{q-1}\right)^{2}+ & \left(\frac{4 q}{1+\varepsilon}-q|q-1| \alpha(1+\varepsilon)\right) \int_{-\infty}^{u} \frac{\xi^{2}}{|t|} d t \\
& \leqq\left(q r^{2}|u|^{q-1}\right)^{2}+\frac{q|q-1|(1+\varepsilon)}{2 \alpha(q+1)} \frac{1}{|u|^{2(q+1)}}
\end{aligned}
$$

taking $\alpha=\frac{4}{|q-1|(1+\varepsilon)^{2}}$ (or any $\alpha>0$ if $q=1$ ) we get

$$
r^{2} \dot{\xi}^{2}+\left(\xi-q r^{2}|u|^{q-1}\right)^{2} \leqq\left(q r^{2}|u|^{q-1}\right)^{2}+\frac{q(q-1)^{2}}{8(q+1)}(1+\varepsilon)^{3} \frac{1}{|u|^{2(q+1)}} ;
$$

hence the estimate $(3.26)$; and

$$
|r \dot{\xi}|=\left.|r| u\right|^{q}-\sqrt{1-\xi^{2}} \mid \leqq\left(q+|q-1| \sqrt{\frac{q}{8(q+1)}}\right) \frac{1}{|u|^{q+1}}(1+o(1)),
$$

but $\sqrt{1-\xi^{2}}-1=0\left(1 /|u|^{2(q+1)}\right)$, hence $(3.27)$.

Suppose now that $q<1$. From (3.5), if there is any $u<0$ such that $\dot{G}(u)=0$, then at this point

$$
\xi / \sqrt{1-\xi^{2}}=-(1-q) r / 2|u|<0,
$$

and if $|u|$ is large enough, $\xi=-\frac{1-q}{2|u|^{q+1}}(1+\circ(1))$. This is impossible from the estimate (3.26), since $c<(1-q) / 2$. Then for large $|u|, G$ is decreasing, negative, and $\xi$ is positive from (3.4). In fact $G$ is decreasing and negative on $(-\infty, a)$, since it is decreasing as long as $\xi>0$ and nonnegative when $\xi=0$. Then $\xi$ is also positive on $(-\infty, a)$ and the curve can be expressed in terms of a function of $r$ on this interval. 
We cannot extend our previous results to the case $N>2$, because the derivative of the function $G$ defined in (1.10) contains the new term - $(N-2) r \xi \dot{\xi}^{2} / \sqrt{1-\xi^{2}}$ which has the sign of $\xi$ and is not necessarily integrable. However, we can give precisely the behaviour of those of the singular solutions which have no vertical point near $-\infty$; we prove also that when $f$ is convex (for example $f$ is a power less than one), such a solution has no vertical point whenever $u<a$ :

Theorem 3.3. Suppose that $N \geqq 2$ and $f$ satisfies

$$
(\ddot{f})^{-} / f^{2} \in L^{1}(-\infty, a) \text {. }
$$

Then:

(i) Any radial local solution $r \rightarrow u(r)$ of $(0.1),(0.2)$ satisfies

$$
\begin{aligned}
& \lim _{r \rightarrow 0} r f(u)=-(N-1), \\
& \lim _{r \rightarrow 0} u^{\prime}(r)=+\infty .
\end{aligned}
$$

(ii) When $\ddot{f}(u) \geqq 0$ on $(-\infty, a), u$ can be extended as a function of $r$ on whole $(0, r(a))$, and satisfies on this interval the estimates

$$
\begin{gathered}
0<\xi=\cos \psi<2 r^{2} \dot{f}(u) /(N-1), \\
-\frac{N+1}{2 \sqrt{N-1}} r \dot{f}(u)<|f(u)|-\frac{N-1}{r}<\frac{r \dot{f}(u)}{\sqrt{N-1}} .
\end{gathered}
$$

(iii) When $f(u)=(N-1)|u|^{q-1} u(q>0)$, then $u$ satisfies near the origin the estimates

$$
\begin{aligned}
0<\xi & \leqq\left(2 q+c^{+}\right) r^{(q+1) / q}(1+\circ(1)), \\
\left.|| u\right|^{q}-1 / r \mid & \leqq \frac{q+c^{+}}{\sqrt{N-1}} r^{1 / q}(1+\circ(1)),
\end{aligned}
$$

where $c^{+}=(q-1)^{+} \sqrt{q / 8(q+1)}$.

Proof. 1st step. By hypothesis the singular solution is a function $r \rightarrow u(r)$ near 0 , which means that $\xi(u)>0$ near $-\infty$. Consider the function $G$ defined in $(1.10)$ by

$$
G(u)=\frac{r^{2}}{2} \dot{\xi}^{2}+\frac{N-1}{2} \xi^{2}-r^{2} \dot{f}(u) \xi ;
$$

from (1.11) we get

$$
\dot{G}(u) \leqq r^{2}(\ddot{f})^{-}(u) \xi .
$$


From (3.31) we can define the function

$$
V(u)=\int_{-\infty}^{u} r^{2}(\ddot{f})^{-}(w) \xi d w
$$

and the function $K=G-V$ is nonincreasing, and bounded near $-\infty$ : from (1.7) (1.9) we have $|r \dot{\xi}(u)| \leqq 2 N-1$ and $\lim _{u \rightarrow-\infty} r^{2} \dot{f}(u)=0$, since $r^{2} \dot{f}(u) \leqq N \dot{f}(u) / f^{2}(u)$. Then $K$ has a finite limit $\ell / 2$ at $-\infty$; hence defining $v$ by (3.10), we get, as in (3.11),

(3.40) $\lim _{u \rightarrow-\infty}\left(r^{2} \dot{\xi}^{2}+(N-1) \xi^{2}\right)=\lim _{v \rightarrow-\infty}\left(\left(\frac{d \xi}{d v}\right)^{2}+(N-1) \xi^{2}\right)=\ell$.

Since $\xi>0$ near $-\infty$, we have $\lim _{v \rightarrow-\infty} \xi=\sqrt{\ell /(N-1)}$ and $\lim _{v \rightarrow-\infty} \frac{d \xi}{d v}=$ 0 ; if $\ell \neq 0$ then $u^{\prime}(r)=\sqrt{1-\xi^{2}} / \xi$ has a finite limit, which is impossible since $u$ is singular. Hence $\ell=0$ and we get (3.33), (3.34) from (1.9).

2nd step. Suppose $\ddot{f}(u) \leqq 0$ on $(-\infty, a)$. From Proposition 1.1 the curve can be extended as a function $r$ of $u$ on $(-\infty, a)$. Here the function $G$ is decreasing near $-\infty$, with $\lim _{u \rightarrow-\infty} G(u)=0$. It remains decreasing and negative and $\xi$ remains positive on $(-\infty, a)$, as at the end of the proof of Theorem 3.2. We get (3.34) from (1.11), and also

$$
\frac{r^{2} \dot{\xi}^{2}}{2}+\frac{N-1}{2}\left(\xi-\frac{r^{2} \dot{f}(u)}{N-1}\right)^{2}<\frac{r^{4} \dot{f}^{2}(u)}{2(N-1)}
$$

hence

$$
|\dot{\xi}|=|| f(u)\left|-\frac{N-1}{r} \sqrt{1-\xi^{2}}\right|<\frac{r \dot{f}(u)}{\sqrt{N-1}} ;
$$

now $\dot{\xi}=-\frac{d}{d r} \sqrt{1-\xi^{2}}$, then, from the convexity of $f$,

$$
\sqrt{1-\xi^{2}}-1<\int_{0}^{r} \frac{\rho \dot{f}(u(\rho)) d \rho}{\sqrt{N-1}} \leqq \dot{f}(u(r)) \frac{r^{2}}{2 \sqrt{N-1}},
$$

hence (3.35).

3rd step. Suppose $f(u)=(N-1)|u|^{q-1} u(q>0)$. Let $\varepsilon>0$. From (1.10), (1.11) and (3.22) we extend (3.28) under the form

$$
\begin{aligned}
\frac{r^{2} \dot{\xi}^{2}}{N-1}+\left(\xi-q r^{2}|u|^{q-1}\right)^{2} & +2 \frac{N-2}{N-1} \int_{-\infty}^{u} \frac{r \xi \dot{\xi}^{2}}{\sqrt{1-\xi^{2}}} d u+\frac{4 q}{1+\varepsilon} \int_{-\infty}^{u} \frac{\xi^{2}}{|t|} d t \\
& \leqq\left(q r^{2}|u|^{q-1}\right)^{2}+2 q(q-1)^{+}(1+\varepsilon) \int_{-\infty}^{u} \frac{|\xi|}{|t|^{q+2}} d t
\end{aligned}
$$


hence we get (3.36) (3.37) as in Theorem 3.2.

Remark 3.3. As in the linear case the estimates (3.36), (3.37) have to be compared to the very accurate estimates concerning the singular solution $U$ constructed in Theorem 2.1: from (2.1) it satisfies

$$
\xi=\cos \psi=\left(1+U^{\prime 2}(r)\right)^{-1 / 2}=q r^{(q+1) / q}\left(1-m r^{2(q+1) / q}(1+\circ(1))\right),
$$

and

$$
|U|^{q}-1 / r=-\mu r^{(q+2) / q}(1+o(1))
$$

where $m=\left((3 N+1) q^{2}+(N+5) q+2\right) / 2(N-1)$ and $\mu=q(q(N+1)+$ 2) $/ 2(N-1)$.

\section{Some global properties.}

Here we give a global existence result extending those of [CF3], [B2], [APS]: we shall suppose that $a=0$ in $(0.3)$, more precisely that

$$
f \text { is increasing on } \mathbb{R} \text { from }-\infty \text { to }+\infty \text {, with } f(0)=0 \text {. }
$$

The assumption (2.3) can be written under the form

$$
f(u) \geqq(N-1) u \dot{f}(u), \quad \text { for any } u<0,
$$

a slightly weaker assumption on $f$ (whenever $f(0)=0$ ) is

$$
(N-1) u f(u) \geqq N F(u), \quad \text { for any } u<0 \text {. }
$$

Theorem 4.1. (i) Under the assumptions (0.4), (4.1), each regular or singular solution of the parametrical system (0.11) can be uniquely extended as a function $u \rightarrow r(u)$ up to a real $\bar{u} \geqq 0$.

(ii) If $\bar{u}=0$, then $\lim _{u \rightarrow 0} r(u)=+\infty$ and $\lim _{u \rightarrow 0} \dot{r}(u)=+\infty$.

(iii) If $\bar{u}>0$, then $F(\bar{u})<2, \bar{r}=\lim _{u \rightarrow \bar{u}} r(u)$ is finite and $\lim _{u \rightarrow 0} \dot{r}(u)=$ $+\infty$.

Let $r_{1}=r(0)$ be the intersection point with the $r$ axis, and $\psi_{1}=\psi(0)$ be the incidental angle. When $\psi_{1} \in[\pi / 2, \pi)$, the curve has a unique minimal point $\widetilde{u} \in(0, \bar{u})$. When $\psi_{1} \in(0, \pi / 2)$, the curve can be expressed as a function $r \rightarrow u(r)$ on $\left[r_{1},+\infty\right)$, with $\lim _{r \rightarrow+\infty} u(r)=\lim _{r \rightarrow+\infty} u^{\prime}(r)=0$.

(iv) Assumption (4.3) implies $\psi_{1}<\pi / 2$. 
(v) Moreover, when $f$ satisfies

$$
q f(u) \geqq u \dot{f}(u), \quad \text { for any } u<0, \quad \text { where } q>1 /(N-1),
$$

then $\psi_{1}$ can be estimated by

$$
\cos \psi_{1}>2((N+q+1)((N-1) q-1))^{1 / 2} / N(q+1) .
$$

Proof. (i) From Proposition 1.1 each solution is defined as a function $r(u)$ at least on $(-\infty, 0)$; now $(0.12)$, equivalent to $(0.11)$ as long as $\sin \psi>0$, can be written as a system

$$
(\dot{r}(u), \dot{\rho}(u))=\left(\rho,(N-1)\left(1+\rho^{2}\right) / r+f(u)\left(1+\rho^{2}\right)^{3 / 2}\right)=\mathbf{H}(u,(r, \rho)),
$$

where $\mathbf{H}$ is continuous in $u$ and $C^{1}$ in $(r, \rho)$ on $\mathbb{R} \times((0,+\infty) \times \mathbb{R})$; then the solution has a unique extension to a maximal interval with upperbound $\bar{u} \geqq 0$. From (1.3) (1.4), $F(u)-\cos \psi$ decreases to a finite limit when $u$ goes to $\bar{u}$, hence $F(u)$ is bounded and $\bar{u}$ is finite; then $\cos \psi$ has a limit, $\dot{r}^{2}$ has a limit $\lambda \in[0,+\infty]$. In fact $\lambda$ is infinite, because of the maximality.

(ii) Suppose $\bar{u}=0$. When $u \in(-1,0)$, we know that $J(u)>J(-1)>$ 0 from (1.5) to (1.7); hence $r^{N-1}>J(-1)\left(1+\dot{r}^{2}\right)$ and $\lim _{u \rightarrow 0} r=+\infty$, $\lim _{u \rightarrow 0} \dot{r}=+\infty, \lim _{r \rightarrow+\infty} \frac{d s}{d r}=1$ and $\lim _{u \rightarrow \bar{u}} s=+\infty$.

(iii) Suppose now $\bar{u}>0$. For any $u \in[0, \bar{u})$ we have $\psi \in(0, \pi)$, since $J(0)>J(-1)>0$. Then

$$
F(\bar{u})-\varepsilon<F(0)-\cos \psi_{1}<1,
$$

hence $\varepsilon=1, \lim _{u \rightarrow \bar{u}} \psi=0, F(\bar{u})<2$ and $\lim _{u \rightarrow \bar{u}} \dot{r}=+\infty$. If $\lim _{u \rightarrow \bar{u}} r=$ $+\infty$, then $\lim _{u \rightarrow \bar{u}}(\dot{J} / J)(u)=(\dot{f} / f)(\bar{u})$, and $J$ is bounded, which is impossible. Then $\bar{r}=\lim _{u \rightarrow \bar{u}} r$ is finite. On $[0, \bar{u})$ the curve $u \rightarrow r(u)$ can only have one minimal point $\widetilde{u}$, from (1.9); it is the case when $\psi_{1} \in[\pi / 2, \pi)$.

Now consider the case $\psi_{1}<\pi / 2$. Then for any $s \geqq s_{1}=s(0)$, the parametrical curve cannot have vertical points, since

$$
-\cos \psi<F(u)-\cos \psi \leqq-\cos \psi_{1}<0,
$$

and the curve can be expressed as a function $r \rightarrow u(r)$ with $r \geqq r_{1}$, since $0<\cos \psi_{1}<\frac{d r}{d s}<1$. Now (0.6), equivalent to (0.11), can be written as`a system

$$
\left(u^{\prime}(r), w^{\prime}(r)\right)=\left(w,-(N-1)\left(1+w^{2}\right) / r+f(u)\left(1+w^{2}\right)^{3 / 2}\right)=\mathbf{K}(r,(u, w))
$$


where $\mathbf{K} \in C^{1}\left((0,+\infty) \times\left(\mathbb{R}^{\star} \times \mathbb{R}\right)\right)$. The solution has a unique extension to a maximal interval $\left[r_{1}, r_{2}\right)$ where $u$ remains positive. First suppose that $r_{2}=+\infty ; \bar{r}$ is the unique extremum of $u$ on $\left[r_{1},+\infty\right)$, then $u$ decreases to a nonnegative limit $\lambda$, and $F(u)-\cos \psi$ has also a finite limit; hence $\lim _{r \rightarrow+\infty} \cos \psi=\lim _{r \rightarrow+\infty} u^{\prime}(r)=0, \lim _{r \rightarrow+\infty} u^{\prime \prime}=-f(\lambda)$ from (0.6), and $\lim _{r \rightarrow+\infty} u(r)=0$; at last $\lim _{r \rightarrow+\infty} s=+\infty$ since $\frac{d s}{d r}>1$, and the parametrical curve is completely described. Now suppose that $r_{2}$ is finite; then $u\left(r_{2}\right)=0$ and $\psi_{2}=\psi\left(r_{2}\right)$ satisfies $\left|\psi_{2}\right|<\psi_{1}$ from (4.8); if $\psi_{2}=0$, then there would be an $r \in\left(\bar{r}, r_{2}\right)$ such that $\left(r^{N-1} u^{\prime} / \sqrt{1+u^{\prime 2}}\right)^{\prime}(r)=0$, which is impossible from (0.5). Near this point, $(0.11)$ is equivalent to $(0.12)$; then from (4.6) we have local existence and uniqueness, and the curve can locally be expressed in terms of $r \rightarrow u(r)$, since $u^{\prime}\left(r_{2}\right) \neq 0$. As above, it has a unique extension to a maximal interval $\left[r_{2}, r_{3}\right)$ where $u$ remains negative. By induction, either $u$ has a unique extension to $\left[r_{1},+\infty\right)$ with a finite number of zeros $r_{1}<r_{2}<\cdots<r_{k}$ and $\lim _{r \rightarrow+\infty} u(r)=\lim _{r \rightarrow+\infty} u^{\prime}(r)=0$; or we can construct an infinite increasing sequence $\left(r_{n}\right)_{n \geqq 1}$ such that $u\left(r_{n}\right)=0$, sign $u(r)=(-1)^{n-1}$ on $\left(r_{n}, r_{n+1}\right)$, and $\left|\psi_{n}\right|=\left|\psi\left(r_{n}\right)\right|>\left|\psi_{n+1}\right|>0$. If $\lim r_{n}=+\infty$ then the curve is completely described for $s \geqq s_{1}$, by the function $r \rightarrow u(r)$ on $\left[r_{1},+\infty\right)$. If $\lim r_{n}=R$ is finite, $u$ has a finite limit, since $u^{\prime}$ is bounded from (4.8); then $\lim _{r \rightarrow R} u=0, \cos \psi$ has also a limit from (1.3) (1.4); then $\lim _{r \rightarrow R} u^{\prime}=0$, since $u^{\prime}\left(r_{n}\right) u^{\prime}\left(r_{n+1}\right)<0$; and the function $u \equiv 0$ is an extension of the solution on $[R,+\infty)$; it is the unique extension from (1.3) (1.4). The curve is again completely described on $\left[s_{1},+\infty\right)$.

(iv) Here we suppose (4.3) and prove that $\psi_{1}<\pi / 2$. If $\psi_{1} \geqq \psi / 2$ then there is a unique maximal point $\widehat{u}<0$ on the curve such that $\dot{r}(u)<0$ on $(\widehat{u}, 0)$ (if $\psi_{1}=\frac{\pi}{2}$, then 0 is a minimal point). Let $\widehat{r}=r(\widehat{u})$; integrating $(0.6)$ between any $r \in\left[r_{1}, \widehat{r}\right]$ and $\widehat{r}$, we get from (1.7)

$$
\begin{aligned}
\widehat{r}^{N-1}-r^{N-1} \sin \psi & =\int_{r}^{\widehat{r}} \rho^{N-1}|f(u)| d \rho<|f(\widehat{u})|\left(\widehat{r}^{N}-r^{N}\right) / N \\
& <\widehat{r}^{N-1}-|f(\widehat{u})| r^{N} / N,
\end{aligned}
$$

which implies

$$
|f(\widehat{u})|<N r^{-1} \sin \psi, \quad \forall r \in\left[r_{1}, \widehat{r}\right] .
$$

Then from (1.3) (1.4),

$$
\begin{aligned}
-F(\widehat{u}) \leqq F(0)- & \cos \psi_{1}-F(\widehat{u}) \\
& =-(N-1) \int_{\widehat{u}}^{0} \frac{\sin \psi}{r} d u<-(N-1) \widehat{u} f(\widehat{u}) / N,
\end{aligned}
$$


which gives a contradiction. Then $\psi_{1}<\pi / 2$.

(v) Suppose that (4.4) holds. Consider the Pohožaev function $\phi_{\delta_{q}, q}$ defined by (1.12) (1.13); it is nonincreasing whenever $u<0$, and its derivative is nonidentically zero. We have $\phi_{\delta_{q}, q}\left(u_{0}\right)=0$ for the regular solution starting at $u_{0}$; and, from (1.7), $\lim \sup _{u \rightarrow-\infty} \phi_{\delta_{q}, q}(u) \leqq \lim _{u \rightarrow-\infty} r^{N}(\delta-\cos \psi)=0$ for any singular solution. Then $\phi_{\delta_{q}, q}(0)<0$, which means $\cos \psi_{1}>\delta_{q}$, that is (4.5).

Remark 4.1. As a consequence of Theorems 3.3 and 4.1, when $f(u)=$ $|u|^{q-1} u$ with $q \leqq 1$, the singular solution $r \rightarrow U(r)$ constructed in Theorem 2.1 has a unique extension as a function of $r$ on whole $(0,+\infty)$, see Fig. 1, 2. The question is opened when $q>1$.

Remark 4.2. When $f$ satisfies (4.4) with a $q \geqq(N+2) /(N-2)$, then for any singular or regular solution we have $\bar{u}=0$ : the curve cannot cross the axis $u=0$. Indeed, at it was proved in [APS] for the regular case, considering $\phi_{1,(N+2) /(N-2)}$ as above, we would get $\cos \psi_{1}>1$ when crossing the axis, which is impossible; see also [B3] for nonradial solutions.

In the general case, the problem is to determine if $u$ is oscillating or not is still opened, even for a power. Let us recall what we know up to now: Suppose $f(u)=|u|^{q-1} u(q \geqq 1 /(N-1))$. From $[\mathbf{P u S}]$, either the solution is oscillating, with an amplitude of the order of $r^{-2(N-1) /(q+3)}$, or keeps an constant sign, and $u=0\left(r^{-2 /(q-1)}\right), u^{\prime}=0\left(r^{-(q+1) /(q-1)}\right)$ at $+\infty$. Since $\lim _{r \rightarrow+\infty} u^{\prime}(r)=0$, equation (0.1) appears as a small perturbation of the equation

$$
\Delta u+|u|^{q-1} u=0
$$

and in fact we can prove that the behaviour near infinity is the same for the two equations: when $u$ keeps a constant sign, either there is a $c>0$ such that $\lim _{r \rightarrow+\infty} r^{N-2} u(r)=c$, or

$$
\lim _{r \rightarrow+\infty} r^{2 /(q-1)} u(r)=-\left(2(N-(N-2) q) /(q-1)^{2}\right)^{1 /(q-1)}
$$

(which implies $q>N /(N-2)$, see [B3]).

When $q \leqq N /(N-2)$ (or $N=2$ ) the curve necessarily oscillates around the axis $u=0$, see [NiS1]. However from (4.5) when $N \geqq 3$ and $q=N /(N-2)$ the incidental angle is quite small: $\psi_{1}<9.6^{\circ}$ when $N=3,4$, and less for larger $N$.

When $N /(N-2)<q<(N+2) /(N-2)$ the question is opened. Numerically it seems that all the curves cross the axis, see Fig. 6,8 . But if it is the case, the angle $\psi_{1}$ is very small from (4.5): $\psi_{1}<3.9^{\circ}$ when $N=3, q=4$, 
$\psi_{1}<1.8^{\circ}$ when $N=3, q=4,5$; and $\psi_{1}$ goes to zero when $q$ increases to $(N+2) /(N-2)$.

Serrin [S2] noticed that regular solutions with $\left|u_{0}\right|$ small enough must oscillate: indeed their slope is small and (0.1) is again a perturbation of (4.10), which has no ground state in this range. We can give a more precise result:

Proposition 4.1. Let $f(u)=|u|^{q-1} u$ with $N /(N-2)<q<(N+2) /(N-2)$. Then any regular solution of $(0.11)$ is oscillating for large $r$, whenever

$$
\left|u_{0}\right|^{q+1} \leqq(q+1)(N+2-(N-2) q) /(N+q+1) .
$$

Proof. Consider the function $\phi_{1, q}$ defined in (1.12):

$$
\dot{\phi}_{1, q}(u)=r^{N}\left(1-\cos \psi+\frac{|u|^{q+1}}{q+1}+\frac{N}{q+1} u \frac{\sin \psi}{r}\right)
$$

then, from (1.13),

$$
\phi_{1, q}(u)=\frac{r^{N-1}}{(q+1) \sin \psi}(N+q+1)(1-\cos \psi)\left(\cos \psi-\frac{(N-1) q-1}{N+q+1}\right) .
$$

Now from (1.3)(1.4), for any $u>u_{0}, F(u)-\cos \psi<F\left(u_{0}\right)-1$. Then (4.14) implies that $\cos \psi>((N-1) q-1) /(N+q+1)$; and $\phi_{1, q}$ is increasing whenever $u<0$. Suppose that $u$ keeps a constant sign; then $u(r)=0\left(r^{-2 /(q-1)}\right)$ and $\sin \psi=0\left(r^{-(q+1) /(q-1)}\right)$ at $+\infty$, hence $\phi_{1, q}(u)=0\left(r^{((N-2) q-(N+2)) /(q-1)}\right)$. Then $\lim _{u \rightarrow 0} \phi_{1, q}(u)=0$, and $\phi_{1, q}\left(u_{0}\right)=0$, which is impossible.

Remark 4.3. The next question is to determine what happens when $f(u)=|u|^{q-1} u$, with $0<q<1 /(N-1)$. Since $1 /(N-1) \leqq 1$ the singular solution $U$ has no vertical point, hence $\psi_{1}<\pi / 2$. On the contrary for regular solutions we can see numerically that $\psi_{1}$ can be greater or lower than $\pi / 2$, depending on the initial data $u_{0}$, or on the value of $q$, see Fig. 1 .

To end this paragraph we give some results about the maximal diameter of the regular curves, and other related questions, which extend [CF3], [F3], [F6]. From Proposition 1.2, the maximal diameter is twice the radius $r_{\alpha}$ of the first maximal point $u_{\alpha}$ under the axis $u=0$ (whenever it exists, that is when $u_{0}<\bar{u}_{0}$ ). For simplification we only consider the case of a power.

Proposition 4.2. Let $f(u)=|u|^{q-1} u$, where $q>0$. Then

(i) There is a uniform bound for the diameter $r_{\alpha}$ of all the regular solutions. 
(ii) On the interval $\left(-\left(q(N-1)^{2} / \sqrt{2 N-1}\right)^{1 /(q+1)}, 0\right)$ any regular solution can have at most one maximal point and one minimal point.

(iii) When $q>1 /(N-1)$ then more precisely

$$
r_{\alpha}<N /\left(\delta_{q}(q+1)(N-1)\right)^{q /(q+1)},
$$

where $\delta_{q}$ is given in (1.14). Moreover, on the interval

$$
\left(-\left(\delta_{q}(q+1)(N-1)\right)^{1 /(q+1)}, 0\right),
$$

any regular solution can have at most one minimal point.

Proof. First suppose that $q>1 /(N-1)$. Consider the function $\phi_{\delta_{q}, q}$ defined in (1.12). It is negative and decreasing. Then at the point $u_{\alpha}$ we have, from $(1.7)$

$$
\delta_{q}+\left|u_{\alpha}\right|^{q+1} /(q+1)<N\left|u_{\alpha}\right| /(q+1) r_{\alpha}<N^{1+1 / q} /(q+1) r_{\alpha}^{1+1 / q},
$$

hence $r_{\alpha}$ is uniformly bounded by $N /\left(\delta_{q}(q+1)\right)^{q /(q+1)}$. More precisely, there is a unique inflexion point $r_{\sigma}=r\left(u_{\sigma}\right)$ on the curve, such that $u_{\sigma} \in\left[u_{\alpha}, 0\right)$ and $\cos \psi_{\sigma} \leqq 0$. At this point, we have $r_{\alpha}\left|u_{\sigma}\right|^{q}=(N-1) \sin \psi_{\sigma}$ from (1.9) and $\phi_{\delta_{q}, q}\left(u_{\sigma}\right)<0$; then

$$
\begin{aligned}
\delta_{q}+\left|\cos \psi_{\sigma}\right|+\left|u_{\sigma}\right|^{q+1} /(q+1)<N\left|u_{\sigma}\right| \sin \psi_{\sigma} /(q+1) r_{\sigma} & \\
& =N\left|u_{\sigma}\right|^{q+1} /(q+1)(N-1),
\end{aligned}
$$

hence

$$
\delta_{q}(q+1)(N-1)<\left|u_{\sigma}\right|^{q+1} \leqq\left|u_{\alpha}\right|^{q+1}
$$

then above $u_{\sigma}$ any regular solution can have at most a minimal point; moreover $r_{\alpha}<N /\left|u_{\alpha}\right|^{q}$, hence we get (4.15).

Now consider the general case. Here we follow the proof of [CF3]. Since $r_{\alpha}\left|f\left(u_{\alpha}\right)\right|>N-1$ and $r\left(u_{0}\right)\left|f\left(u_{0}\right)\right|=0$, there is a unique point $r_{\gamma}=r\left(u_{\gamma}\right)$ on the curve, such that $u_{\gamma} \in\left(u_{0}, u_{\alpha}\right), r_{\gamma}\left|f\left(u_{\gamma}\right)\right|=N-1$, and $\sin \psi>0$ on $\left(u_{\gamma}, u_{\alpha}\right)$; then on this interval $r|f(u)|>N-1$, and there is no inflexion point, from (1.9), hence $\psi>\psi_{\gamma}$. Consider the positive increasing function $J$ defined in (1.5): we have $J\left(u_{\gamma}\right)>0$, and consequently

$$
\sin \psi_{\gamma}>(N-1) / N \text {. }
$$

But on $\left(u_{\gamma}, u_{\alpha}\right)$ the curve lies under the "basis curve", hence at the crossing point, $\operatorname{tg} \psi_{\gamma}<\left|u_{\gamma}\right|^{q+1} / q(N-1)$. Then from (4.17),

$$
q(N-1)^{2} / \sqrt{2 N-1}<\left|u_{\gamma}\right|^{q+1},
$$


and (ii) follows. Moreover $r_{\gamma}=(N-1) /\left|u_{\gamma}\right|^{q}$, then

$$
r_{\gamma}<R_{N, q}=\left((N-1)^{1-1 / q} \sqrt{2 N-1} / q\right)^{q /(q+1)} .
$$

Moreover from (1.5)(1.6),

$$
J\left(u_{\alpha}\right)=J\left(u_{\gamma}\right)+q \int_{r_{\gamma}}^{r_{\alpha}} \frac{r^{N}}{N}|u|^{q-1} \operatorname{tg} \psi d r,
$$

but $J\left(u_{\alpha}\right)=r_{\alpha}^{N-1}-r_{\alpha}^{N}\left|u_{\alpha}\right|^{q} / N<r_{\alpha}^{N-1} / N$, and $\operatorname{tg} \psi>\operatorname{tg} \psi_{\gamma}$ on $\left(r_{\alpha}, r_{\gamma}\right)$; then from (4.17),

$$
r_{\alpha}^{N-1} \geqq q \frac{N-1}{\sqrt{2 N-1}} \int_{r_{\gamma}}^{r_{\alpha}} r^{N}|u|^{q-1} d r
$$

on this interval, we have $N-1<r|u|^{q}<N$, hence from (4.19)

$$
r_{\alpha}^{N-1} \geqq c_{N, q}\left(r_{\alpha}^{N+1 / q}-R_{N, q}^{N+1 / q}\right),
$$

where $c_{N, q}=q(N-1)^{2-1 / q} /(N+1 / q) \sqrt{2 N-1}$ when $q \geqq 1$, and $c_{N, q}=$ $q(N-1) N^{1-1 / q} /(N+1 / q) \sqrt{2 N-1}$ when $q<1$. And $(4.20)$ implies an uniform bound for $r_{\alpha}$.

Remark 4.4. From (4.15) we establish that for large $q$, the maximal diameter of the curves is small, which could be foreseen numerically, see Fig. $4,5,7$.

\section{Appendix A. The fixed point theorem.}

Proof of Theorem 2.1. It is the direct generalization of the proof of [CF1]. Let

$$
c=(q(N+1)+2) / 2(N-1), \quad \text { and } k=2+1 / q .
$$

We write equation (2.1) as a system of unknown $\left(u, v, \alpha_{0}, \alpha_{1}\right)$ :

$$
\left\{\begin{array}{l}
u(r)=-r^{-1 / q}+\left(c+\alpha_{0}(r)\right) r^{k} \\
v(r)=q^{-1} r^{-(q+1) / q}+k\left(c+\alpha_{1}(r)\right) r^{k-1}
\end{array}\right.
$$

(3)

$$
\left\{\begin{array}{l}
\alpha_{1}(r)=\alpha_{0}(r)+k^{-1} r \alpha_{0}^{\prime}(r), \\
v^{\prime}(r)=-(N-1)\left(|u(r)|^{q-1} u(r)\left(1+v^{2}(r)\right)^{3 / 2}+r^{-1} v(r)\left(1+v^{2}(r)\right)\right) .
\end{array}\right.
$$


We write it under the form

(4)

$$
\left\{\begin{array}{l}
\alpha_{1}(r)=\alpha_{0}(r)+r \alpha_{0}^{\prime}(r) / k \\
r^{-\beta}\left(r^{k}\left(r^{\beta} \alpha_{0}(r)\right)^{\prime}\right)^{\prime}+\gamma^{2} r^{-k} \alpha_{0}(r)=\mathbf{F}(r),
\end{array}\right.
$$

where we choose

$$
\gamma=\sqrt{N-1} / q, \quad \beta=N / 2+2(k-1) ;
$$

and $\mathbf{F}$ is given in function of $\alpha_{0}, \alpha_{1}$ by

$$
\text { (6) } \begin{aligned}
\mathbf{F}(r)= & (k-1) q^{-1} r^{-k}-k(k-1) c r^{k-2}+(\beta-1)(\beta-k) \alpha_{0}(r) r^{k-2} \\
& +k(2 \beta-k) \alpha_{1}(r) r^{k-2}+\gamma^{2} \alpha_{0}(r) r^{-k} \\
& -(N-1)\left(|u(r)|^{q-1} u(r)(1+v(r))^{3 / 2}+v(r)\left(1+v^{2}(r)\right) r^{-1}\right),
\end{aligned}
$$

where $u, v$ are defined by (2). Now introduce the kernel of equation (3): set $\alpha=\left(\alpha_{0}, \alpha_{1}\right)$. Then the system takes the form

$$
\alpha=\mathbf{T}(\alpha)=\left(\mathbf{T}_{0}(\alpha), \mathbf{T}_{1}(\alpha)\right),
$$

where

$$
\mathbf{T}_{0}(\alpha)=\gamma^{-1} r^{-\beta} \int_{0}^{r} \tau^{\beta} \mathbf{F}(\tau) \sin \frac{\gamma}{k-1}\left(\tau^{1-k}-r^{1-k}\right) d \tau,
$$

$\mathbf{T}_{1}(\alpha)=\left(1-\beta k^{-1}\right) \mathbf{T}_{0}(\alpha)+k^{-1} r^{1-\beta-k} \int_{0}^{r} \tau^{\beta} \mathbf{F}(\tau) \cos \frac{\gamma}{k-1}\left(\tau^{1-k}-r^{1-k}\right) d \tau$.

Let $\sigma \in(0,2(k-1))$ be fixed. For any $M>0$ and any $R \in(0,1]$ we set

$$
\begin{aligned}
B_{M, R}=\left\{\alpha=\left(\alpha_{0}, \alpha_{1}\right) \in\right. & C^{0}\left([0, R], \mathbb{R}^{2}\right) \mid\|\alpha\| \\
& \left.=\max _{r \in(0, R]}\left|r^{-\sigma} \alpha_{0}(r)\right|+\max _{r \in[0, R]}\left|\alpha_{1}(r)\right| \leqq M\right\} .
\end{aligned}
$$

We are going to prove that for any $M>0$, there is an $R=R(M)$ such that $\mathbf{T}$ is a strict contraction of $B_{M, R(M)}$.

Notice first that for $R(M)$ small enough, $u(r)$ is negative for any $\alpha \in$ $B_{M, R(M)}$. Then let us compute

$$
\begin{aligned}
\mathbf{F}(r)= & (k-1) q^{-1} r^{-k}-k(k-1) c r^{k-2}+(\beta-1)(\beta-k) \alpha_{0}(r) r^{k-2} \\
& +k(2 \beta-k) \alpha_{1}(r) r^{k-2}+\gamma^{2} \alpha_{0}(r) r^{-k}+r^{2-3 k} \mathbf{H}\left(\alpha(r), r^{2(k-1)}\right),
\end{aligned}
$$


where

$\mathbf{H}(\alpha, t)=(N-1) q^{-3}(1-p t)^{q}\left(1+m t+s^{2} t^{2}\right)^{3 / 2}-(1+s t)\left(1+m t+s^{2} t^{2}\right)$,

with

$$
p=c+\alpha_{0}, \quad m=q\left(2 k c+q+2 k \alpha_{1}\right), \quad s=q k\left(c+\alpha_{1}\right) .
$$

Then we develop $\mathbf{H}$ up to the order 3 in $t$ and obtain

$$
\begin{aligned}
\mathbf{H}\left(\alpha, r^{2(k-1)}\right)= & (N-1) q^{-3}(m / 2-q p-s) r^{2(k-1)} \\
+ & (N-1) q^{-3}\left(s^{2}+3 m^{2} / 4-3 m q p+q(q-1) p^{2}-2 m s\right) \\
& \cdot r^{4(k-1)} / 2+r^{6(k-1)} Q(\alpha, r),
\end{aligned}
$$

where, for $R(M)$ small enough, for any $\alpha \in B_{M, R(M)}$ and $r \in[0, R(M)]$,

$$
|Q(\alpha(r), r)|+\left|\frac{\partial Q}{\partial \alpha_{0}}(\alpha(r), r)\right|+\left|\frac{\partial Q}{\partial \alpha_{1}}(\alpha(r), r)\right| \leqq C(M),
$$

and $C(M)$ depends only on $M ;(15)$ comes from suitable estimates of $\frac{\partial^{3} \mathbf{H}}{\partial t^{3}}$, $\frac{\partial^{4} \mathbf{H}}{\partial \alpha_{0} \partial t^{3}}, \frac{\partial^{4} \mathbf{H}}{\partial \alpha_{1} \partial t^{3}}$. When using (14) in (11), with our choice of parameters $\gamma, c$ and $\beta$, the coefficients of $r^{-k}, \alpha_{0} r^{-k}, \alpha_{1} r^{k-2}$ vanish, so that we get

$$
\mathbf{F}(r)=\lambda r^{k-2}+P(\alpha(r)) r^{k-2}+Q(\alpha(r), r) r^{3 k-4},
$$

where

$$
\lambda=-k(k-1) c+(N-1)\left(3 q / 4+(k-3) c-6 q^{-1} k c^{2}+(q-1) q^{-2} c^{2}\right) / 2,
$$

$$
P(\alpha)=\mu \alpha_{0}-3(N-1) q^{-1} k \alpha_{0} \alpha_{1}+(N-1)(q-1) q^{-2} \alpha_{0}^{2} / 2,
$$

$$
\mu=(\beta-k)(\beta-1)+(N-1) q^{-2}\left(2(q-1) c-3\left(2 k q c+q^{2}\right)\right) / 2 .
$$

Now we can estimate the right terms of (8) (9). For any function $\phi$ on 
$(0,+\infty)$ we set

(20)

(21)

$$
\mathbf{S}(\phi)(r)=\int_{0}^{r} \phi(\tau) \sin \frac{\gamma}{k-1}\left(\tau^{1-k}-r^{1-k}\right) d \tau
$$

$$
\mathbf{C}(\phi)(r)=\int_{0}^{r} \phi(\tau) \cos \frac{\gamma}{k-1}\left(\tau^{1-k}-r^{1-k}\right) d \tau,
$$

and get for any real $\rho>-k$ the relations

$$
\begin{aligned}
& \mathbf{C}\left(r^{\rho}\right)=\gamma^{-1}(\rho+k) \mathbf{S}\left(r^{\rho+k-1}\right) \\
& \mathbf{S}\left(r^{\rho}\right)=\gamma^{-1}(\rho+k)-\gamma^{-2}(\rho+2 k-1)(\rho+k) \mathbf{S}\left(r^{\rho+2(k-1)}\right) .
\end{aligned}
$$

They imply the estimates

$$
\begin{aligned}
& \mathbf{S}\left(r^{\rho}\right)=\gamma^{-1} r^{\rho+k}+0\left(r^{\rho+3 k-2}\right) \\
& \mathbf{C}\left(r^{\rho}\right)=\gamma^{-2}(\rho+k) r^{\rho+2 k-1}+0\left(r^{\rho+4 k-3}\right) .
\end{aligned}
$$

Then

$$
\begin{aligned}
\gamma^{-1} r^{-\beta} \mathbf{S}\left(\lambda r^{\beta+k-2}\right) & =\gamma^{-2} \lambda r^{2(k-1)}+0\left(r^{4(k-1)}\right), \\
k^{-1} r^{1-\beta-k} \mathbf{C}\left(\lambda r^{\beta+k-2}\right) & =\gamma^{-2} k^{-1}(\beta+2 k-2) \lambda r^{2(k-1)}+0\left(r^{4(k-1)}\right) ;
\end{aligned}
$$

moreover from (10) and (15) we deduce the following estimates, with new constants $C(M)$ :

$$
\begin{aligned}
\left|\mathbf{S}\left(P(\alpha) r^{\beta+k-2}\right)\right|+\left|\mathbf{C}\left(P(\alpha) r^{\beta+k-2}\right)\right| & \leqq C(M) r^{\beta+k-1+\sigma}, \\
\left|\mathbf{S}\left(Q(\alpha, r) r^{\beta+3 k-4}\right)\right|+\left|\mathbf{C}\left(Q(\alpha, r) r^{\beta+3 k-4}\right)\right| & \leqq C(M) r^{\beta+3(k-1)} .
\end{aligned}
$$

Then for any $\alpha \in B_{M, R(M)}$,

$$
\|\mathbf{T}(\alpha)\| \leqq C(M)\left(R(M)^{2(k-1)-\sigma}+R(M)^{k-1}+R(M)^{\sigma}\right),
$$

hence $\mathbf{T}$ applies $B_{M, R(M)}$ into itself for $R(M)$ small enough. Then we estimate $\|\mathbf{T}(\alpha)-\mathbf{T}(\widehat{\alpha})\|$ for any $\alpha, \widehat{\alpha} \in B_{M, R(M)}$. From (10) and (15) we get

$$
\begin{aligned}
& \left|\mathbf{S}\left(\left(\alpha_{0}-\widehat{\alpha}_{0}\right) r^{\beta+k-2}\right)\right|+\left|\mathbf{C}\left(\left(\alpha_{0}-\widehat{\alpha}_{0}\right) r^{\beta+k-2}\right)\right| \leqq C(M) r^{\beta+k-1+\sigma}\|\alpha-\widehat{\alpha}\| \\
& \left|\mathbf{S}\left(\left(\alpha_{0} \alpha_{1}-\widehat{\alpha}_{0} \widehat{\alpha}_{1}\right) r^{\beta+k-2}\right)\right|+\left|\mathbf{C}\left(\left(\alpha_{0} \alpha_{1}-\widehat{\alpha}_{0} \widehat{\alpha}_{1}\right) r^{\beta+k-2}\right)\right| \\
& \quad \leqq C(M) r^{\beta+k-1+\sigma}|| \alpha-\widehat{\alpha} \| \\
& \left|\mathbf{S}\left(\left(\alpha_{0}^{2}-\widehat{\alpha}_{0}^{2}\right) r^{\beta+k-2}\right)\right|+\left|\mathbf{C}\left(\left(\alpha_{0}^{2}-\widehat{\alpha}_{0}^{2}\right) r^{\beta+k-2}\right)\right| \leqq C(M) r^{\beta+k-1+2 \sigma}\|\alpha-\widehat{\alpha}\| \\
& \left|\mathbf{S}\left((Q(\alpha, r)-Q(\widehat{\alpha}, r)) r^{\beta+3 k-4}\right)\right|+\left|\mathbf{C}\left((Q(\alpha, r)-Q(\widehat{\alpha}, r)) r^{\beta+3 k-4}\right)\right| \\
& \quad \leqq C(M) r^{\beta+3(k-1)}\|\alpha-\widehat{\alpha}\|
\end{aligned}
$$


hence

$$
\|\mathbf{T}(\alpha)-\mathbf{T}(\widehat{\alpha})\| \leqq C(M)\left(R(M)^{k-1}+R(M)^{\sigma}\right)\|\alpha-\widehat{\alpha}\|,
$$

and $\mathbf{T}$ is a strict contraction of $B_{M, R(M)}$ for $R(M)$ small enough. Consequently it has a unique fixed point $\alpha$. From (6) (8), $\alpha_{0}=\mathbf{T}_{0}(\alpha)$ is in fact in $C^{\infty}([0, R(M)])$, so that the function

$$
U(r)=-r^{-1 / q}+\left(c+\alpha_{0}(r)\right) r^{2+1 / q},
$$

where $c$ is given by (1), is a solution in $(0, R(M)]$ of equation (2.1), which ends the proof.

Remark. With (16) we get the third term in the expansion of $U$ (and the others, as in [CF1]):

$$
U(r)=-r^{-1 / q}+c r^{2+1 / q}+\lambda \gamma^{-2} r^{4+3 / q}(1+\circ(r)),
$$

where $\gamma, \lambda$ are given by (5), (17).

\section{Appendix B. Unduloids in dimension $N$.}

The unduloids are rotationally invariant surfaces of constant positive mean curvature $H$, without any double points and periodic in $u$. Hence they are given by the periodic solutions $u \rightarrow r(u)$ of the equation

$$
\frac{\ddot{r}}{\left(1+\dot{r}^{2}\right)^{3 / 2}}-\frac{N-1}{r} \frac{1}{\sqrt{1+\dot{r}^{2}}}=-N H .
$$

By integration we get

$$
\frac{r^{N-1}}{\sqrt{1+\dot{r}^{2}}}-H r^{N}=A
$$

where necessarily $0<A<(N-1)^{N-1} H^{1-N} / N^{N}$. Set

$$
A=\frac{(N-1)^{N-1}}{N^{N}} H^{1-N}\left(1-k^{2}\right)^{N / 2},
$$

where $k \in(0,1)$. Denote by $\psi$ the angle between a tangent to the curve and the $r$ axis, hence $\cos \psi=\dot{r} / \sqrt{1+\dot{r}^{2}}$. Then from (1) (2), it is easy to verify that $k$ is the maximal value of $|\cos \psi|$ on a periodic loop, attained at the point $r=\sqrt{1-k^{2}}(N-1) / N H$. 
Let $T=T(H, k)$ be the smallest period of the unduloid $\Omega_{k}$ with parameter $k$.
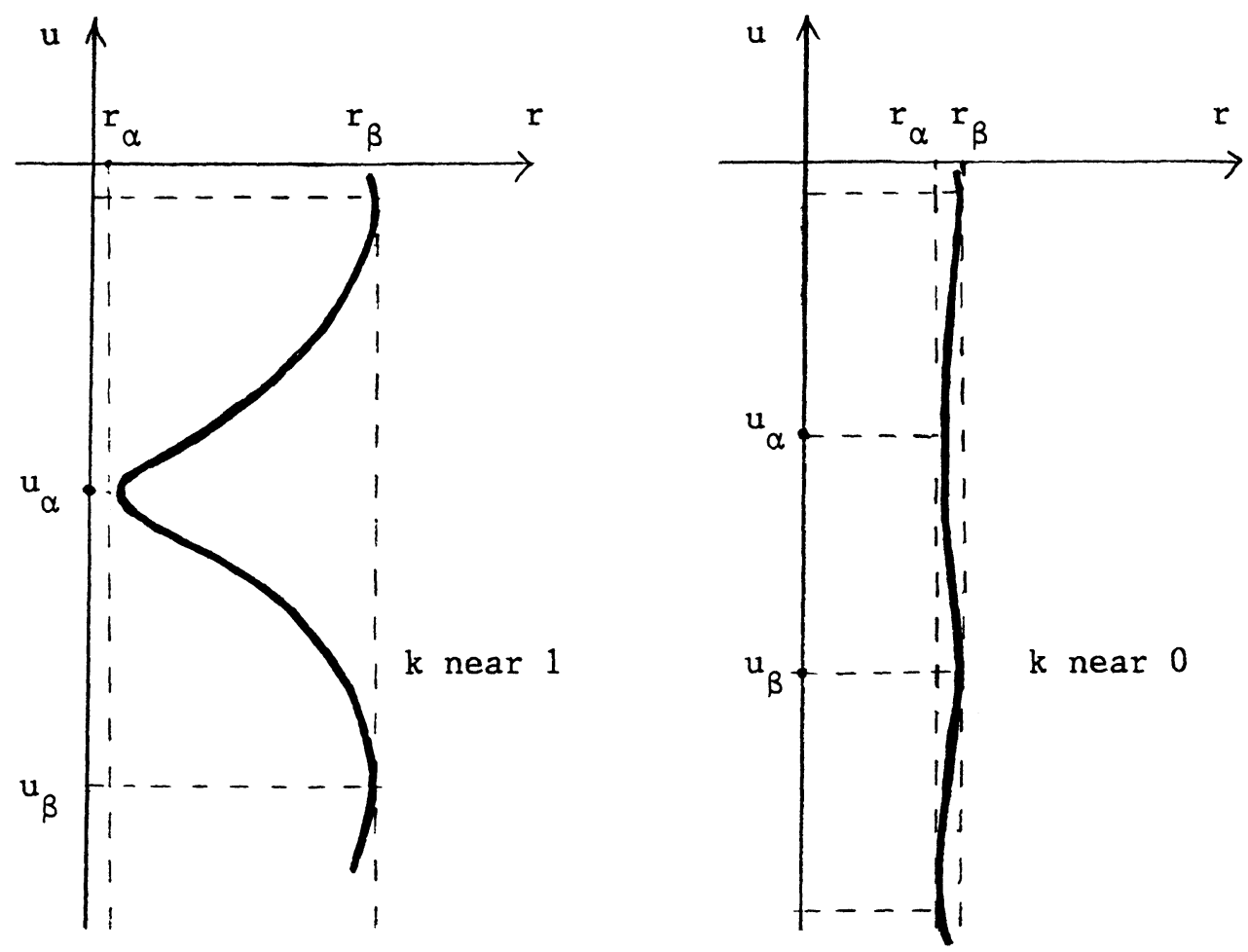

Then from (2) the curve is symmetric by respect to $u=\bar{u}+T / 2$ where $\bar{u}$ is any extremal point, and $T$ is given explicitely in terms of an hyperelliptic integral:

$$
T=2 \int_{r_{\alpha}}^{r_{\beta}}\left(\left(H r+A r^{1-N}\right)^{-2}-1\right)^{-1 / 2} d r
$$

where $r_{\alpha}, r_{\beta}$ are two extremal points, the two solutions of the equation $\mathrm{Hr}+$ $A r^{1-N}=1$. In order to study the period function $T$ we use another way: when $N=2$ any unduloid is the curve traced by the focal point of an ellipse when it is rolled on the $u$-axis. Hsiang and Yu extended this classical result to the dimension $N$ in [HY]: for any unduloid $\Omega$ there exists unique polar coordinate graph $\Gamma, \theta \rightarrow \rho=f(\theta)$, such that $\Omega$ is the trace of the origin by rolling $\Gamma$ on the $u$-axis. Then $\Gamma$ is a periodic curve of period $\tau, T$ is equal to the arc length of $\Gamma$ between $\theta=0$ and $\theta=\tau$, and the extremals of $r$ and $p$ are the same. From $[\mathbf{H Y}]$, Theorem 3 , there is a $\beta>N^{2}(H /(N-1))^{2-2 / N}$ such that

$$
\left(\frac{d \theta}{d w}\right)^{2}=\beta(w-H)^{2 / N}-w^{2}, \quad \text { where } w=1 / r
$$


Then we get

$$
\begin{aligned}
T & =2 \int_{0}^{\tau / 2}\left(\rho^{2}+\left(\frac{d \rho}{d \theta}\right)^{2}\right)^{1 / 2} d \theta \\
& =2 \int_{\rho_{\alpha}}^{\rho_{\beta}}\left(1-\beta^{-1} \rho^{2 / N-2}(1-H \rho)^{-2 / N}\right)^{-1 / 2} d \rho
\end{aligned}
$$

But $\rho_{\alpha}=r_{\alpha}, \rho_{\beta}=r_{\beta}$ are the solutions of the equation $\rho^{2-2 / N}(1-H \rho)^{2 / N}=$ $\beta^{-1}$, hence $\beta=A^{-2 / N}$, and we get a second formula for $T$ :

$$
T=2 \int_{r_{\alpha}}^{r_{\beta}}\left(1-A^{2 / N} r^{2 / N-2}(1-H r)^{-2 / N}\right)^{-1 / 2} d r .
$$

Now we can prove the following lemma, which extends the results of [F5] to any dimension $N$ :

Lemma B1. The period function of an unduloid $\Omega_{k}$ with curvature $H$ and parameter $k$ is given by

$$
T(H, K)=I(k) / H
$$

where $I$ is a continuous positive function on $[0,1]$.

Proof. For any $k \in(0,1)$, we make the change of variables $r=(N-1)(1+$ t) $/ \mathrm{NH}$ in formula (6), and get (7), with

$$
I(k)=\frac{2(N-1)}{N} \int_{t_{\alpha}(k)}^{t_{\beta}(k)}\left(1-\frac{1-k^{2}}{K(t)}\right)^{-1 / 2} d t,
$$

where

$$
K(t)=(1+t)^{2-2 / N}(1-(N-1) t)^{2 / N},
$$

and $t_{\alpha}(k), t_{\beta}(k)$ are the two solutions of the equation $K(t)=1-k^{2}$, with $-1<t_{\alpha}(k)<0<t_{\beta}(k)<1 /(N-1)$.

Now the two functions $t_{\alpha}, t_{\beta}$ are differentiable in $(0,1)$, and we can write, as in $[\mathbf{B B}]$,

$$
\begin{aligned}
\frac{N}{2(N-1)} I(k) & =\int_{0}^{k} \frac{d}{d q}\left(t_{\beta}-t_{\alpha}\right)(q)\left(1-\frac{1-k^{2}}{1-q^{2}}\right)^{-1 / 2} d t \\
& =\int_{0}^{1} \frac{d}{d q}\left(t_{\beta}-t_{\alpha}\right)(k \tau) \sqrt{1-k^{2} \tau^{2}} \frac{d \tau}{\sqrt{1-\tau^{2}}} .
\end{aligned}
$$


Then $I$ is a continuous function on $(0,1)$, obviously positive. We have to prove that $I$ has positive limits at 0 and 1 . Let for any $q \in(0,1)$,

$$
W(q)=\frac{d}{d q}\left(t_{\beta}-t_{\alpha}\right)(q) \sqrt{1-q^{2}}=2 q\left(\frac{1}{K^{\prime}\left(t_{\alpha}(q)\right)}-\frac{1}{K^{\prime}\left(t_{\beta}(q)\right)}\right) \sqrt{1-q^{2}},
$$

then $W$ is nonnegative. When $N=2, W(q)=2 \sqrt{1-q^{2}}$ and we find again the result of [CF3], [F5]: $I(k)=2 E(k)$, where $E(k)=\int_{0}^{\pi / 2} \sqrt{1-k^{2} \sin ^{2} \omega} d \omega$; and $I$ is decreasing from $\pi$ to 2 on $[0,1]$. Now look at the case $N>$ 2 : we get easily $\lim _{q \rightarrow 0} q^{-1} t_{\beta}(q)=-\lim _{q \rightarrow 0} q^{-1} t_{\alpha}(q)=1 / \sqrt{N-1}$, hence $\lim _{q \rightarrow 0} W(q)=2 / \sqrt{N-1}$. Now we have $\lim _{q \rightarrow 1} t_{\alpha}(q)=-1, \lim _{q \rightarrow 1} t_{\beta}(q)=$ $1 /(N-1)$, then $\lim _{q \rightarrow 1}\left(1 / K^{\prime}\left(t_{\beta}(q)\right)\right)=0$ since $N>2$. We get

$$
\lim _{q \rightarrow 1} 2(1-q)^{-1 / 2(N-1)} W(q)=N^{(N-2) /(N-1)} /(N-1),
$$

hence $\lim _{q \rightarrow 1} W(q)=0$. The function $W$ is bounded on $(0,1)$ and we can apply the Lebesgue theorem, since $\left(1-\tau^{2}\right)^{-1 / 2}$ is integrable. We obtain $\lim _{k \rightarrow 1} I(k)=2$ and $\lim _{k \rightarrow 0} I(k)=\pi / \sqrt{N-1}$.

Remark. From (5), Hsiang and Yu had given in $[\mathbf{H}, \mathbf{Y}]$ an upper bound for $T$, which means that $I(k) \leqq 2(\pi+1)$. Here we prove that $I$ has also a positive lower bound in $(0,1)$. Incidentally one could expect the form (7) of function $T$, since the change of variables $r(u)=\frac{N-1}{N H} s(v), v=\frac{N H}{N-1} u$ reduces equation (1) into the equation of an unduloid of curvature $N-1$ :

$$
\frac{d^{2} s / d v^{2}}{\left(1+(d s / d v)^{2}\right)^{3 / 2}}-\frac{N-1}{s} \frac{1}{\sqrt{1+(d s / d v)^{2}}}=-(N-1)
$$

hence $T(H, k)=\frac{N-1}{N H} T(N-1, k)$.

In the following lemma we construct an unduloid of comparison in dimension $N$, whose curvature is given implicitely, as in [F5], [I] when $N=2$.

Lemma B2. Let $b<a, v_{\alpha}<\beta$ and $r_{\alpha}$ be small enough: such that

$$
0<r_{\alpha}<(N-1) / \mid f\left(v_{\alpha}-N M / 2|f(b)|\right), \quad \text { where } \quad M=\max _{k \in[0,1]} I(k) .
$$

Then there exists an unduloid $\Omega_{k}$ with consecutive extremal points $\left(v_{\alpha}, r_{\alpha}\right)$, $\left(v_{\beta}, r_{\beta}\right)$, with $v_{\beta}<v_{\alpha}, r_{\beta}>r_{\alpha}$ and curvature $\left|f\left(v_{\beta}\right)\right| / N$. Its parameter $k$ is given by the relation

$$
k^{2}=1-K\left(-1+r_{\alpha}\left|f\left(v_{\beta}\right)\right| /(N-1)\right)
$$


where $K$ is defined in (9).

Proof. If $\Omega_{k}$ exists, its period is given by

$$
T(k)=2\left(v_{\alpha}-v_{\beta}\right)=N I(k) /\left|f\left(v_{\beta}\right)\right| .
$$

For any $k \in[0,1]$ there is a unique $v(k)<v_{\alpha}$ such that

$$
2\left(v_{\alpha}-v(k)\right)=N I(k) /|f(v(k))| .
$$

Indeed the continuous function $v \rightarrow y(v)=2\left(v_{\alpha}-v\right)+N I(k) / f(v)$ is decreasing from $(0.3)$, and $\lim _{v \rightarrow-\infty} y(v)=+\infty$ and $y\left(v_{\alpha}\right)<0$. Moreover $v(k)>v_{\alpha}-N I(k) / 2|f(b)|$; then from $(12), t_{\alpha}=-1+r_{\alpha} \mid f(v(k) \mid /(N-1)<0$. Consequently $r_{\alpha}$ is a minimal point of $\Omega_{k}$ if and only if $K\left(t_{\alpha}\right)=1-k^{2}$. Let us define for any $k \in[0,1]$,

$$
\Theta(k)=\left(1-K\left(r_{\alpha}|f(v(k))| /(N-1)-1\right)\right)^{1 / 2} .
$$

Then from Lemma B1, $\Theta$ takes $[0,1]$ continuously into itself, and has a fixed point $\bar{k}$, and $\Omega_{\bar{k}}$ satisfies Lemma B2. 


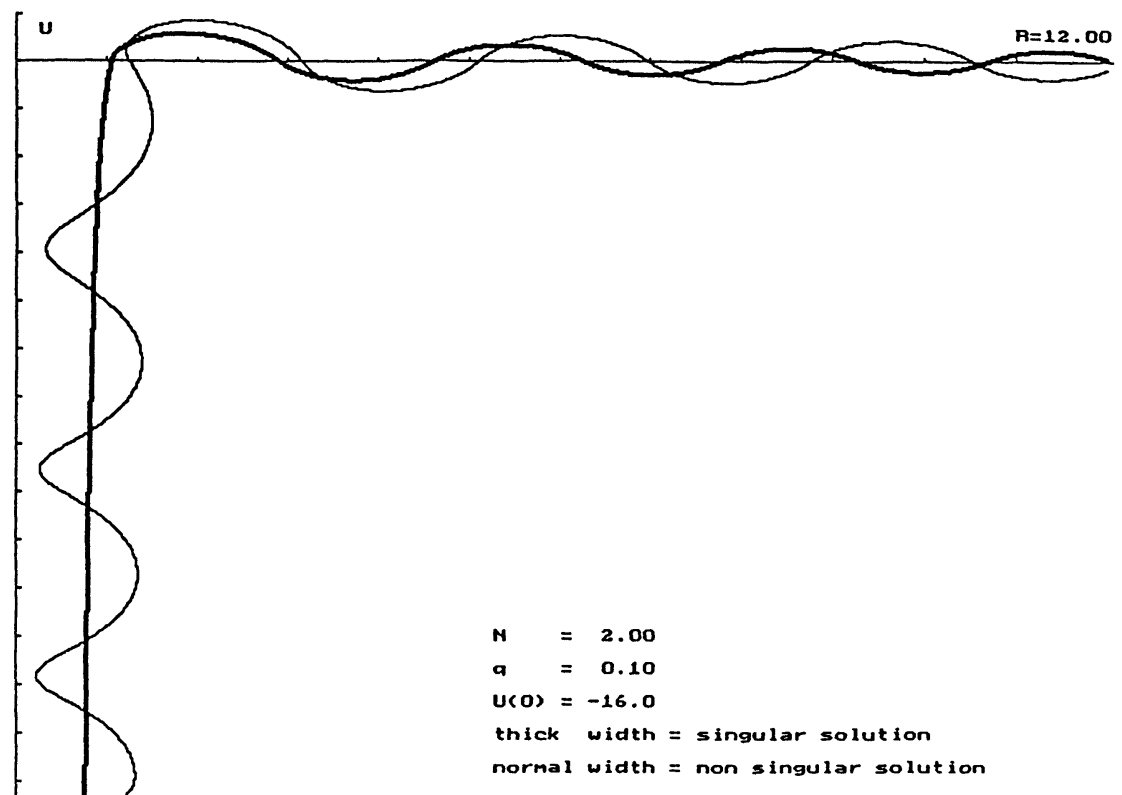

Figure 1.

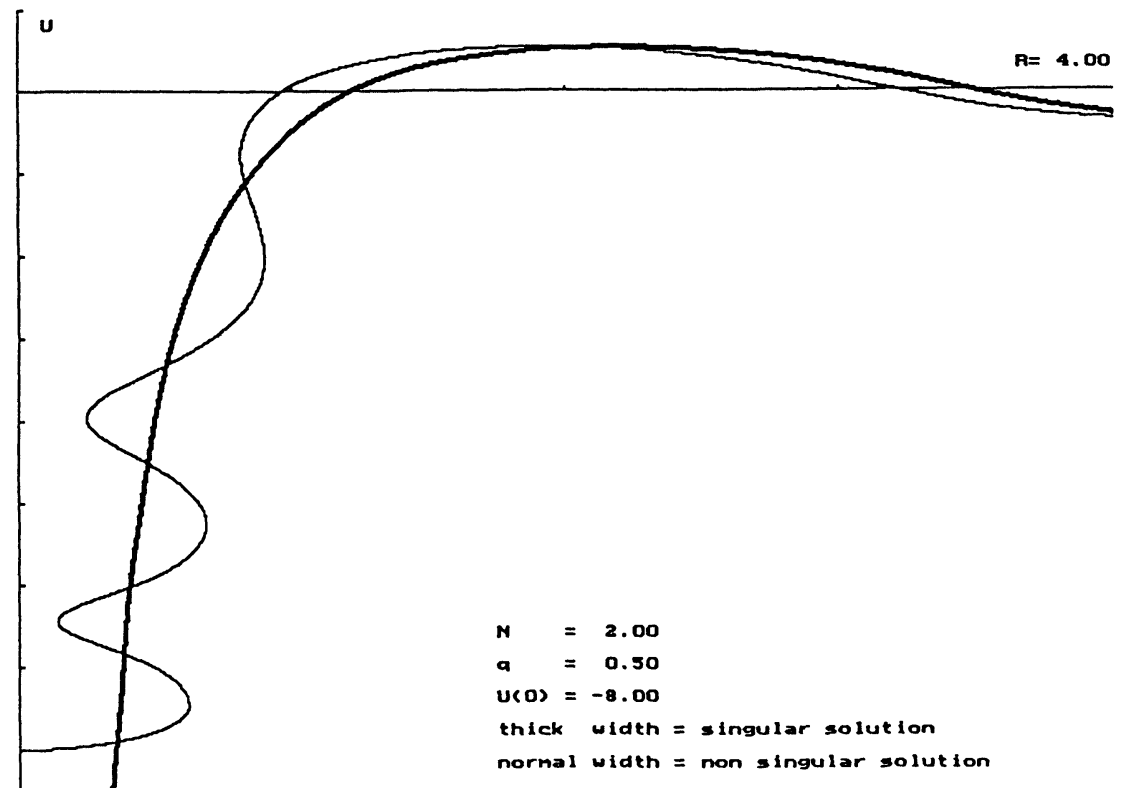

Figure 2. 


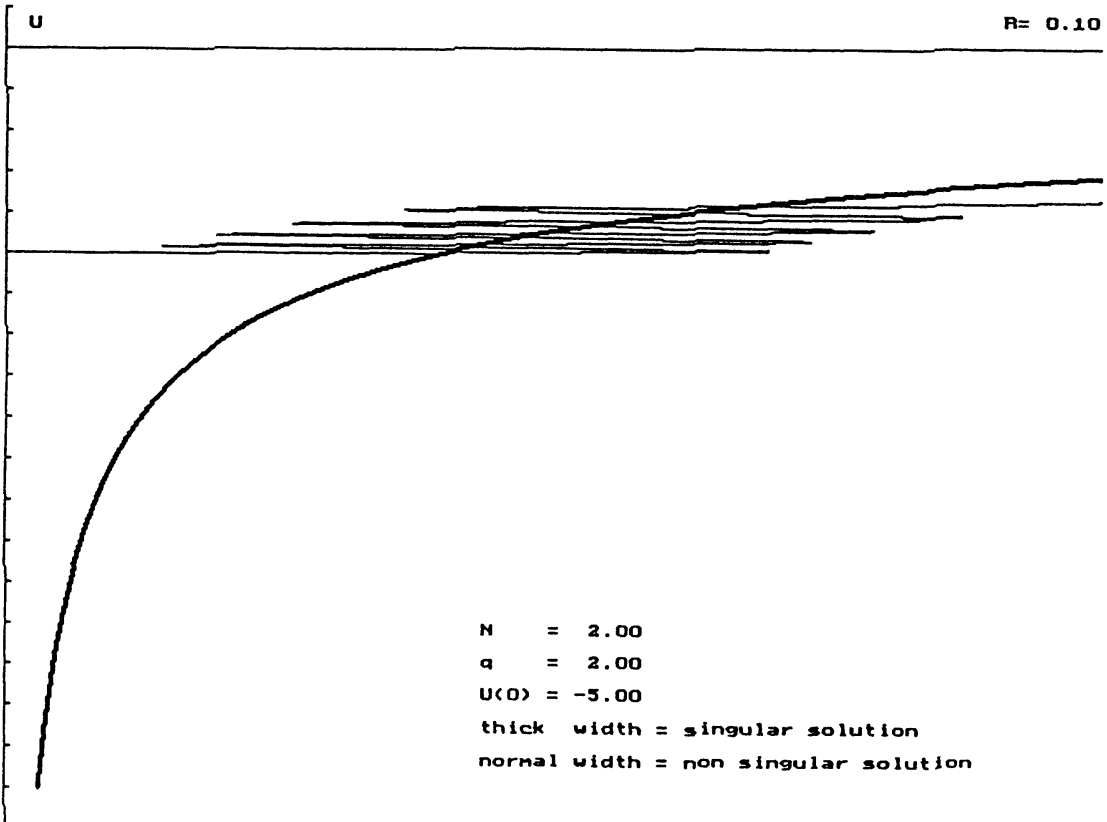

Figure 3.

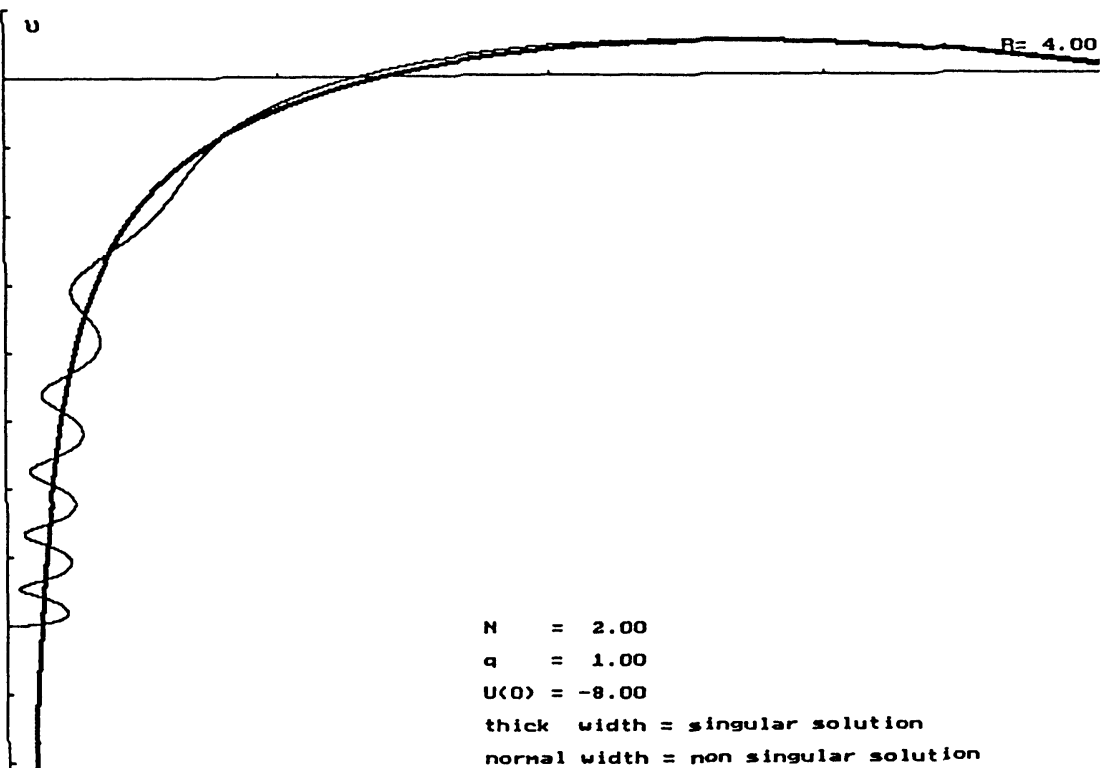

Figure 4. 


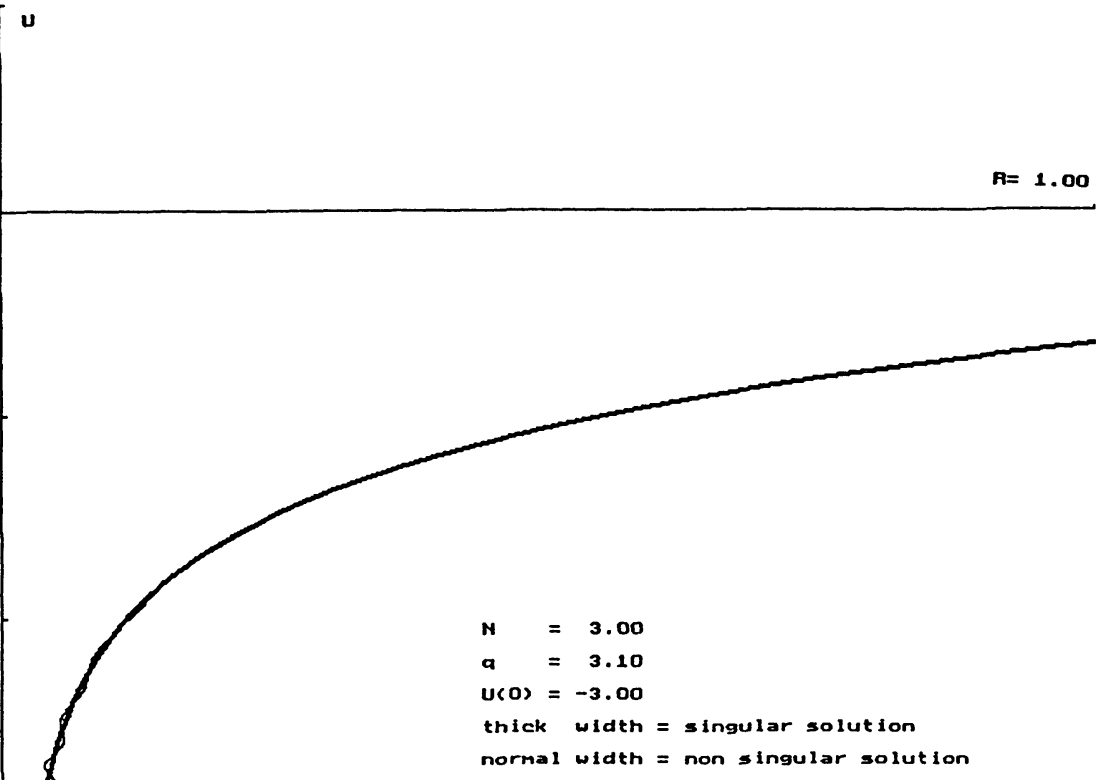

Figure 5.

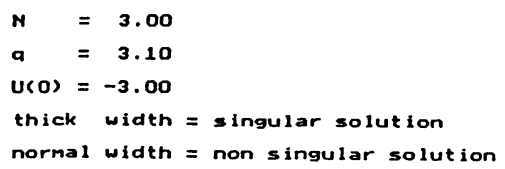

Figure 6. 
$u$

$\mathrm{A}=0.10$

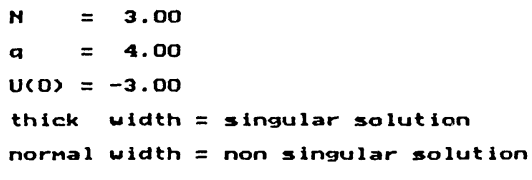

Figure 7.

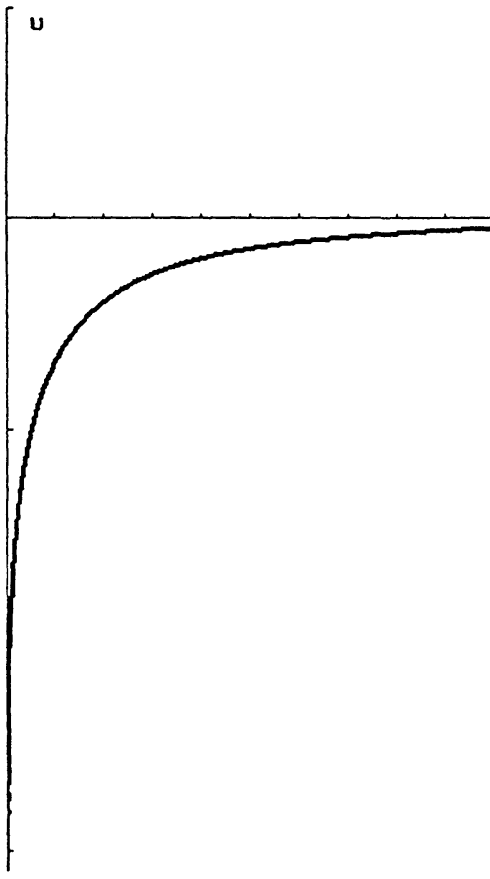

$R=24.00$

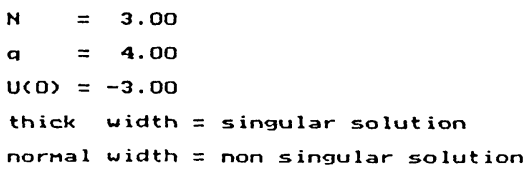

Figure 8. 


\section{References}

[A] F.V. Atkinson, Self-intersecting solutions of the prescribed mean curvature equation, Argonne Report ANL-87-26, 4 (1989), 1-20.

[AP] F.V. Atkinson and L.A. Peletier, Bounds for vertical points for prescribed mean curvature equations, I, Proc. Roy. Soc. Edinburg, 112 (1989), 15-32.

[APS] F.V. Atkinson, L.A. Peletier, and J. Serrin, Ground states for the prescribed mean curvature equation: the supercritical case, Nonlinear diffusion equations and their equilibrium states, Springer-Verlag, (1988), 51-73.

[B1] M.F. Bidaut-Veron, New results concerning the singular solutions of the capillarity equation, Variational methods for free surfaces interfaces, Springer-Verlag, (1985), 191-196.

[B2]

[BB] M.F. Bidaut-Veron and M. Bouhar, On characterization of solutions of some nonlinear differential equations and applications, Siam. J. of Math. Anal., 25(3) (1994), 859-875.

[CF1] P. Concus and R. Finn, A singular solution of the capillarity equation, I. Existence, Invent. Math., 29 (1975), 143-148.

[CF2] , A singular solution of the capillarity equation, II. Uniqueness, Invent. Math., 29 (1975), 149-160.

[CF3] - The shape of a pendent liquid drop, Philos. Trans. Roy. Soc. London, A, 292 (1979), 307-340.

[F1] R. Finn, On the behavior of a capillary surface at a singular point, J. Anal. Math., 30 (1976), 156-163.

[F2] - Some properties of capillarity free surfaces, Sem. on minimal submanifolds, Princeton Univ. Press, (1983), 323-337.

, On the pendent liquid drop, Zeit Anal. Anwend., 4 (1985), 331-339.

[F4] _ Equilibrium capillary surfaces, Springer-Verlag, 1986.

[F5] - Green's identities and pendent liquid drops, I, New Developments in Part. Diff. Eq. and Appl. to Math. Physics, Ferrara, Plenum Press, 1991.

[F6] _ Green's identities and pendent liquid drops, II, Proc. Symp. Cont. Mech. and Related Problems Anal., Tbilissi, 1991.

[FLS] B. Franchi, E. Lanconelli, and J. Serrin, Existence and Uniqueness of positive solutions of quasilinear equations in $\mathbb{R}^{n}$, Accad. Naz. Lincei, 79 (1989), 121-126.

[HY] W.Y. Hsiang and W.C. Yu, A generalization of a theorem of Delaunay, J. Diff. Geometry, 16 (1981), 161-177.

[I] N. Ishimura, Nonlinear eigenvalue problem associated with the generalized capillarity equation, J. Fac. Sc. Univ. Tokyo, 37 (1990), 457-466.

[NiS1] W.N. Ni and J. Serrin, Existence and nonexistence theorems for ground states of quasilinear partial differential equations: the anomalous case, Accad. Naz. Lincei, 77 (1986), 231-257. 
ential equations, Comm. on Pure and Appl. Math., 34 (1986), 379-399.

[PS] L.A. Peletier and J. Serrin, Ground states for the prescribed mean curvature equation, Proc. A.M.S., 100(4) (1987), 694-700.

[PuS] P. Pucci and J. Serrin, Continuation and limit properties for solutions of strongly nonlinear second order differential equations, Asymptotic Anal., 4 (1991), 97-160.

[S1] J. Serrin, Positive solutions of a prescribed mean curvature problem, Lecture Notes Math., Springer-Verlag, 1340 (1989), 248-255.

[S2] , Personal communication.

[W1] H.C. Wente, The symmetry of sessile and pendent drops, Pac. J. of Math., 88(2) (1980), 387-397.

[W2] - The stability of the axially symmetric pendent drop, Pac. J. of Math., 88(2) (1980), 421-470.

Received July 20, 1993 and revised December 10, 1993.

UNIVERSite DE TOURS

PARC DE GRANDMONT

37200 TOURS FRANCE 



\title{
PACIFIC JOURNAL OF MATHEMATICS
}

Founded by

\author{
$\begin{array}{ll}\text { E. F. Beckenbach (1906-1982) F. Wolf (1904-1989) } & \text { F }\end{array}$
}

EDITORS

Sun-Yung A. Chang (Managing Editor) Robert Finn

University of California

Los Angeles, CA 90095-1555

pacific@math.ucla.edu

F. Michael Christ

University of California

Los Angeles, CA 90095-1555

christ@math.ucla.edu

Nicholas Ercolani

University of Arizona

Tucson, AZ 85721

ercolani@math.arizona.edu
Stanford University

Stanford, CA 94305

finn@gauss.stanford.edu

Steven Kerckhoff

Stanford University

Stanford, CA 94305

spk@gauss.stanford.edu

Martin Scharlemann

University of California

Santa Barbara, CA 93106

mgscharl@math.ucsb.edu
Gang Tian

Massachusettes Institute of Technology

Cambridge, MA 02139

tian@math.mit.edu

\section{S. Varadarajan}

University of California

Los Angeles, CA 90095-1555

vsv@math.ucla.edu

Dan Voiculescu

University of California

Berkeley, CA 94720

dvv@math.berkeley.edu

\section{SUPPORTING INSTITUTIONS}

\author{
CALIFORNIA INSTITUTE OF TECHNOLOGY \\ NEW MEXICO STATE UNIVERSITY \\ OREGON STATE UNIVERSITY \\ STANFORD UNIVERSITY \\ UNIVERSITY OF ARIZONA \\ UNIVERSITY OF BRITISH COLUMBIA \\ UNIVERSITY OF CALIFORNIA \\ UNIVERSITY OF HAWAII
}

\author{
UNIVERSITY OF MONTANA \\ UNIVERSITY OF NEVADA, RENO \\ UNIVERSITY OF OREGON \\ UNIVERSITY OF SOUTHERN CALIFORNIA \\ UNIVERSITY OF UTAH \\ UNIVERSITY OF WASHINGTON \\ WASHINGTON STATE UNIVERSITY
}

The supporting Institutions listed above contribute to the cost of publication of this Journal, but they are not owners or publishers and have no responsibility for its contents or policies.

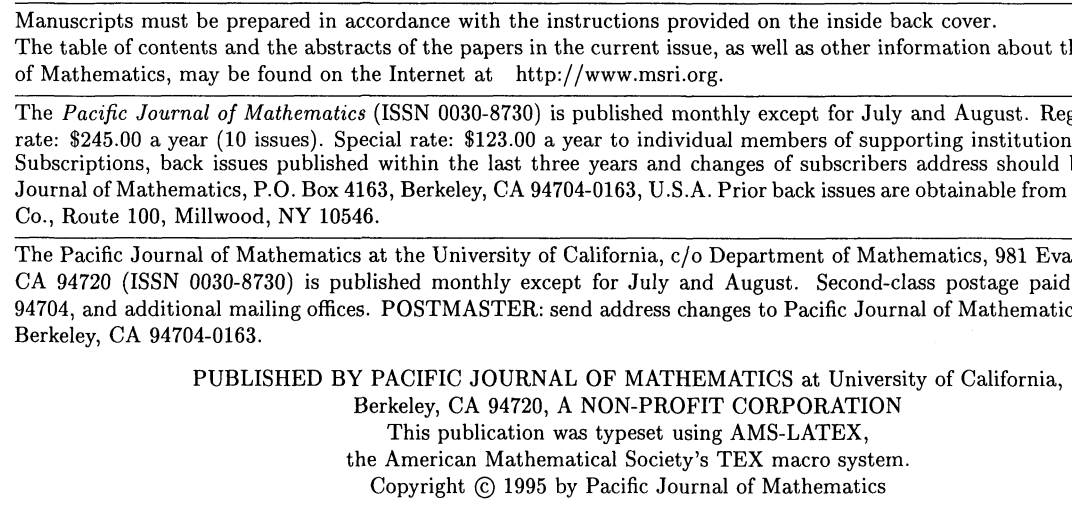




\section{PACIFIC JOURNAL OF MATHEMATICS}

\section{Volume $173 \quad$ No. $1 \quad$ March 1996}

Isometric immersions of $H_{1}^{n}$ into $H_{1}^{n+1}$

KINETSU ABE

Rotationally symmetric hypersurfaces with prescribed mean curvature

MARIE-FRANÇOISE BIDAUT-VÉRON

The covers of a Noetherian module

JIAN-JUN CHUAI

On the odd primary cohomology of higher projective planes

MARK FoSKEY and MiCHAEL DAVID SLACK

Unit indices of some imaginary composite quadratic fields. II

MiKinito HiRABAYASHI

Mixed automorphic vector bundles on Shimura varieties

MIN Ho LeE

Trace ideal criteria for Toeplitz and Hankel operators on the weighted Bergman

spaces with exponential type weights

PENG LIN and RICHARD ROCHBERG

On quadratic reciprocity over function fields

KATHY DONOVAN MERRILL and LYNNE WALLING

$\left(A_{2}\right)$-conditions and Carleson inequalities in Bergman spaces

TAKAHIKO NAKAZI and MASAHIRO YAMADA

A note on a paper of E. Boasso and A. Larotonda: "A spectral theory for solvable Lie 173 algebras of operators"
C. Отт

Tensor products with anisotropic principal series representations of free groups

Carlo Pensavalle and Tim Steger

On Ricci deformation of a Riemannian metric on manifold with boundary

YING SHEN

The Weyl quantization of Poisson $S U(2)$

Albert Jeu-Liang Sheu

Weyl's law for $S L(3, \mathbb{Z}) \backslash S L(3, \mathbb{R}) / S O(3, \mathbb{R})$

ERIC GeORge Stade and Dorothy IRENe Wallace (ANDREOli)

Minimal hyperspheres in two-point homogeneous spaces

PER TOMTER

Subalgebras of little Lipschitz algebras

NiKOLAI ISAAC WEAVER 